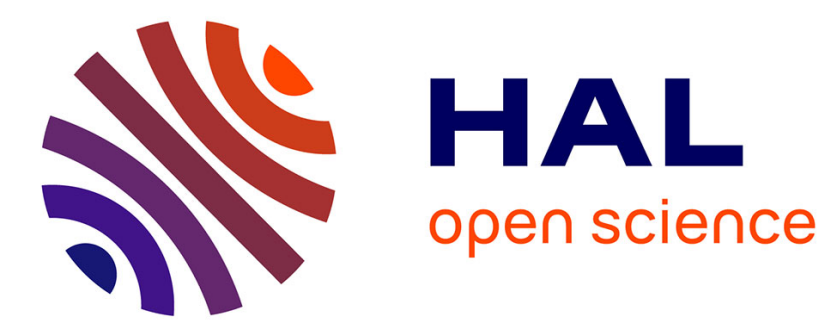

\title{
Robust Asymptotic Stabilization of Nonlinear Systems With Non-Hyperbolic Zero Dynamics
}

Lorenzo Marconi, Laurent Praly, Alberto Isidori

\section{To cite this version:}

Lorenzo Marconi, Laurent Praly, Alberto Isidori. Robust Asymptotic Stabilization of Nonlinear Systems With Non-Hyperbolic Zero Dynamics. IEEE Transactions on Automatic Control, 2010, 55 (4), pp.907-921. 10.1109/TAC.2010.2042001 . hal-00531495

HAL Id: hal-00531495

https://hal-mines-paristech.archives-ouvertes.fr/hal-00531495

Submitted on 2 Nov 2010

HAL is a multi-disciplinary open access archive for the deposit and dissemination of scientific research documents, whether they are published or not. The documents may come from teaching and research institutions in France or abroad, or from public or private research centers.
L'archive ouverte pluridisciplinaire HAL, est destinée au dépôt et à la diffusion de documents scientifiques de niveau recherche, publiés ou non, émanant des établissements d'enseignement et de recherche français ou étrangers, des laboratoires publics ou privés. 


\title{
Robust Asymptotic Stabilization of Nonlinear Systems With Non-Hyperbolic Zero Dynamics
}

\author{
Lorenzo Marconi, Member, IEEE, Laurent Praly, Member, IEEE, and Alberto Isidori, Fellow, IEEE
}

\begin{abstract}
We present a general tool to handle the presence of zero dynamics which are asymptotically but not locally exponentially stable in problems of robust nonlinear stabilization by output feedback. We show how it is possible to design locally Lipschitz stabilizers under conditions which only rely upon a partial detectability assumption on the controlled plant, by obtaining a robust stabilizing paradigm which is not based on design of observers and separation principles. The main design idea comes from recent achievements in the field of output regulation and specifically in the design of nonlinear internal models.
\end{abstract}

Index Terms-Minimum-phase systems, output feedback, robust stabilization, uniform completely observable (UCO).

\section{INTRODUCTION}

$\mathbf{T}$ HE problem of output feedback stabilization, in the large, of nonlinear systems has been a subject of intensive investigation in the past twenty years or so (for a partial summary of the literature see, e.g., [15]). Initially, most of the contributions addressed the design of memoryless full state-feedback or partial state-feedback stabilizing laws, for specific classes of nonlinear systems. In this respect, it is worth mentioning the methods for global stabilization via full state-feedback of systems in lower-triangular form, based on recursive design [3] and back-stepping [10], [18], and for stabilization with guaranteed region of attraction (that is, "semi-global" stabilization) via partial state-feedback of systems possessing a globally asymptotically stable zero dynamics ([2], [3]), based on high-gain. Then, the attention of the investigators shifted to the study of dynamic output feedback stabilization schemes, mainly intended to obtain stability with guaranteed region of attraction, as a reasonable alternative to global stability, for which certain obstructions had been in the meanwhile identified (see [25]). Among the various research directions proposed in this setting, a special role is played by the nonlinear separation principle, the well-established (in a linear context) principle of replacing the full state-feedback by an asymptotic estimate, provided by an appropriate observer (see [29]). The main limitation of this approach, though, is the possible lack of robustness of the resulting

Manuscript received December 27, 2008; revised May 25, 2009 and May 25, 2009. First published February 02, 2010; current version published April 02, 2010. Recommended by Associate Editor S. Celikovsky.

L. Marconi is with C.A.SY.-DEIS, University of Bologna, Bologna 40126, Italy (e-mail: lorenzo.marconi@unibo.it).

L. Praly is with École des Mines de Paris, Fontainebleau, Paris 75272, France (e-mail: Laurent.Praly@ensmp.fr).

A. Isidori is with DIS, Università di Roma "La Sapienza," Rome 00185, Italy (e-mail: albisidori@dis.uniroma1.it).

Digital Object Identifier 10.1109/TAC.2010.2042001 controller, a byproduct of the intrinsic difficulties associated with the design of robust state observers for nonlinear systems. Furthermore, full state observability of the controlled plant is not, in principle, a necessary condition for output feedback stabilization. A major step forward to overcome these limitations has been taken in [28], with the introduction of the concept of Uniform Completely Observable (UCO) control law, namely a state-feedback law which can be expressed as a (known) nonlinear function of the control input, the measured output, and their time derivatives. If a system possesses a UCO stabilizing law, the issue of full-state estimation is replaced by the more tractable issue of estimating a number of higher derivatives of input and output. This, in [28], has been achieved by a mix of back-stepping and partial-state observation techniques, yielding a dynamic output feedback stabilizer which is robust to the extent in which the UCO stabilizing law is not affected by uncertainties and is vanishing at the desired equilibrium. It is important to stress, though, that in the stabilization scheme of [28], asymptotic convergence is guaranteed only if the given UCO stabilizing law is in turn locally exponentially stabilizing. Practical stability must be accepted otherwise (see also [7] in this regard). The latter limitation may be overtaken with the design of a local nonlinear observer in the spirit of [29], by resorting again to a nonlinear separation principle. However, in so-doing, the concerns regarding the possible lack of robustness come forward again.

The hypothesis that a properly defined "subsystem" possesses a (locally) exponentially stable equilibrium is recurrent in several problems of asymptotic analysis and design of nonlinear systems. This is the case in the analysis of singularly perturbed systems ([9], [31]), where the so-called boundary layer system is required to possess an exponentially stable attractor, in the method of averaging ([26], [27], [31]), where the so-called averaged system is required to be locally exponentially stable, in the design of feedback stabilizing laws for systems possessing a globally asymptotically stable zero dynamics ("minimum-phase" nonlinear systems [3], [15]).

In this paper we present a tool to handle the presence of not necessarily hyperbolic zero dynamics in the problem of stabilizing nonlinear systems by means of output feedback (see also [19] for alternative approaches based on time-varying output feedback). As particular application, this tool is then used to extend the main stabilization results of [28], overtaking in this way the obstruction caused by the lack of exponential stability in the back-stepping procedure and in the design of the robust observer. More specifically, by means of the tools which have been developed in a context of nonlinear output regulation (see [6], [22], [24]), we show how the design of a dynamic output feedback control law which asymptotically stabilizes a compact 
attractor can be obtained by starting from a UCO state-feedback control law which does not necessarily stabilize in exponential way the desired asymptotic attractor and which is not necessarily vanishing on it. We will show that these limitations can be removed by adding an extra dynamics whose role is to compensate the effect of certain undesired interconnections terms which are not vanishing on the desired asymptotic attractor and which, as a consequence, can not be dominated only by means of high-gain. This will enable us to set up a dynamic back-stepping algorithm and an extended partial-state observer algorithm which embed solution techniques typical of internal model-based design.

This work is organized as follows. In the next section the design framework and the general result are given. Section III, partitioned in three subsections, is focused on the application of the proposed tool in the context of the stabilization tools presented in [28]. Then, Section IV presents a few conditions, obtained by mild adaptation of results proposed in the output regulation literature, useful to construct the dynamic regulator which solves the problem discussed in Section II. Finally Sections V and VI conclude with examples and final remarks.

Notation: For $x \in \mathbb{R}^{n},|x|$ denotes the Euclidean norm and, for $\mathcal{C}$ a closed subset of $\mathbb{R}^{n},|x|_{\mathcal{C}}=\min _{y \in \mathcal{C}}|x-y|$ denotes the distance of $x$ from $\mathcal{C}$. For $\mathcal{S}$ a subset of $\mathbb{R}^{n}, \operatorname{cl} \mathcal{S}$ and int $\mathcal{S}$ are the closure of $\mathcal{S}$ and the interior of $\mathcal{S}$ respectively, and $\partial \mathcal{S}$ its boundary. A continuous function $\gamma: \mathbb{R} \rightarrow \mathbb{R}$ is said to be class $\mathcal{K}$ if $\gamma(0)=0$ and it is strictly increasing. A continuous function $\beta: \mathbb{R}_{+} \times \mathbb{R} \rightarrow \mathbb{R}_{+}$is said to be class- $\mathcal{K} \mathcal{L}$ if for all $t \in \mathbb{R}_{+}$the function $\beta(t, \cdot)$ is class- $\mathcal{K}$ and for all $s \in \mathbb{R}$, the function $\beta(\cdot, s)$ is strictly decreasing and $\lim _{t \rightarrow \infty} \beta(t, s)=0$. A class- $\mathcal{K} \mathcal{L}$ function $\beta(\cdot, \cdot)$ satisfying $|s| \leq d \Rightarrow \beta(t, s) \leq$ $N e^{-\lambda t}|s|$ for some positive $d, N, \lambda$ is said to be a locally exponential class- $\mathcal{K} \mathcal{L}$ function. For a locally Lipschitz system of the form $\dot{z}=f(z)$ the value at time $t$ of the solution passing through $z_{0}$ at time $t=0$ will be written as $\phi_{f}\left(t, z_{0}\right)$ or, if the initial condition and the system are clear from the context, as $z(t)$ or $z\left(t, z_{0}\right)$. For a smooth system $\dot{x}=f(x), x \in \mathbb{R}^{n}$, a compact set $\mathcal{A}$ is said to be $\operatorname{LAS}(\mathcal{X})$ (respectively $\operatorname{LES}(\mathcal{X})$ ), with $\mathcal{X} \subset \mathbb{R}^{n}$ a compact set, if it is locally asymptotically (respectively exponentially) stable with a domain of attraction containing $\mathcal{X}$. By $\mathcal{D}(\mathcal{A})$ we denote the domain of attraction of $\mathcal{A}$ if the latter is LAS/LES for a given dynamics. For a function $f: \mathbb{R}^{n} \rightarrow \mathbb{R}^{n}$ and a differentiable real-valued function $q: \mathbb{R}^{n} \rightarrow \mathbb{R}, L_{f} q(x)$ denotes the Lie derivative at $x$ of $q$ along $f$. For a smooth system $\dot{x}=f(x), x \in \mathbb{R}^{n}$, the $\omega$-limit set of a subset $B \subset \mathbb{R}^{n}$, written $\omega(B)$, is the set of all points $x \in \mathbb{R}^{n}$ for which there exists a sequence of pairs $\left(x_{k}, t_{k}\right)$, with $x_{k} \in B$ and $t_{k} \rightarrow \infty$ as $k \rightarrow \infty$, such that $\lim _{k \rightarrow \infty} \phi_{f}\left(t_{k}, x_{k}\right)=x$.

\section{THE MAIN TOOL}

\section{A. A Motivating Example and Intuition of the Result}

In order to illustrate the main idea developed in the paper, consider the simple 2-D system

$$
\begin{aligned}
& \dot{x}=-x^{3}+w y \\
& \dot{y}=x+u
\end{aligned}
$$

with state $(x, y)$, control input $u$ and measured output $y$, and the problem of stabilizing, in a semiglobal sense, the origin of (1) by means of output feedback (see also [20]). The term $w$ is a non negative constant uncertain parameter taking values in a known compact set. The peculiarity of the previous system is that the origin of its zero dynamics, described by $\dot{x}=-x^{3}$, is globally asymptotically stable but not locally exponentially due to the cubic nonlinearity. For this specific example it is a well-known fact (see [15]) that the absence of local exponential stability of the zero dynamics makes impossible to solve the problem at hand by means of static high-gain output feedback of the form $v=-\kappa y$, where $\kappa>0$ is a design parameter. As a matter of fact, the resulting closed-loop system, obtained by controlling (1) with $v=-\kappa y$, is immediately seen to have three equilibria at $(x, y)=(0,0),(x, y)=\left(\kappa \sqrt{w / \kappa^{3}}, \sqrt{w / \kappa^{3}}\right)$ and $(x, y)=\left(-\kappa \sqrt{w / \kappa^{3}},-\sqrt{w / \kappa^{3}}\right)$ which clearly shows how only semiglobal and practical, in the parameter $\kappa$, stability can be eventually achieved. This limitation, though, can be overcome by adopting smooth dynamic output feedback. Among the different methods which might be successfully used to this purpose, the idea which is followed in this paper is to approach the problem by formulating an appropriate set stabilization problem which, for the considered academic example, can be explained as follow.

Consider the Lyapunov function $V(x)=x^{2}$ associated to the origin of the zero dynamics and the sub-level set $\mathcal{R}:=\{x \in \mathbb{R}$ : $V(x) \leq \epsilon\}$, where $\epsilon$ is a positive number, which contains $x=0$ in its interior. By using elementary Lyapunov arguments, the set $\mathcal{R}$ is clearly seen to be forward invariant for the zero dynamics of (1) and reached in finite time by any trajectory $x(t)$ originating from compact sets of initial conditions. These facts, in turn, can be used to compute a bound on the trajectory $x(t)$ of the form $|x(t)|_{\mathcal{R}} \leq N e^{-\lambda t}|x(0)|_{\mathcal{R}}$, for some positive $N$ and $\lambda$, from which it is immediately concluded that the set $\mathcal{R}$ is LES for the zero dynamics. Thus, for the specific example, the existence of a locally asymptotically stable equilibrium point for the zero dynamics automatically implies the existence of a set, containing the origin, which is LES and which can be taken arbitrarily "small" and "close" to the origin by taking the parameter $\epsilon$ sufficiently small. Not surprisingly this result can be generalized to a general class of nonlinear systems having locally asymptotically attractors (see the forthcoming Lemma 1). With this result at hand, the idea now is to approach the problem of stabilizing the set $\mathcal{R} \times\{0\}$, instead of the origin, of system (1) by taking advantage from the fact that $\mathcal{R}$ is LES and thus that "conventional" high-gain arguments could be effectively used. The vision behind this strategy is that, once asymptotic stability of the set $\mathcal{R} \times\{0\}$ has been eventually established by an appropriate choice of the control law, standard omega-limit set arguments, using the fact that $x=0$ is LAS for the zero dynamics, might be appropriately used to prove the desired asymptotic convergence to the origin of the overall state. This intuition, in turn, can be made precise in a general setting as detailed throughout the paper (see the second part of the proof of next Theorem 2).

The challenging aspect in the formulated set stabilization problem is that the "coupling term" $x$ in the $\dot{y}$ equation in (1) is not vanishing on $\mathcal{R}$, namely the set $\mathcal{R} \times\{0\}$ is not forward invariant for (1) with $u=0$. As a consequence, the problem at 
hand can not be approached only by means of a static control law of the form $u=-\kappa y$ but a dynamic controller is needed. More specifically, the goal of the controller is to compensate for the coupling term $x$ which, indeed, should be reconstructed and not simply dominated by the control law. In this respect the stabilizer is required to have the ability of reproducing, with its output, all the signals $-x(t)$ generated by the zero dynamics $\dot{x}=-x^{3}$ closed to the origin (namely on the set $\mathcal{R}$ ). This feature makes the problem at hand extremely related to problems of output regulation (see [5]) where the controller is precisely required to posses the ability of generating all the (not zero) "steady state" control signals which are needed to enforce a zero regulated error by thus making invariant a compact set on which regulation objectives are met. By inheriting the commonly used terminology of output regulation, it is possible to state that, for the specific problem at hand, the lack of exponential stability in the zero dynamics can be overtaken by embedding in the stabilizer an internal model of the zero dynamics closed to the attractor (the origin for the specific example). It turns out that for the addressed example the dynamic stabilizer having the above-mentioned properties is a controller of the form

$$
\begin{aligned}
& \dot{\eta}=-\operatorname{sat}\left(\eta^{3}\right)-L(\eta+\kappa y) \\
& u=-\kappa y-\eta
\end{aligned}
$$

where $\operatorname{sat}(\cdot)$ is a saturation function (whose saturation level can be taken arbitrary), and $L$ and $\kappa$ are high-gain design parameters to be tuned according to the compact set of initial conditions and of the uncertainty. Details on how the previous controller can be obtained will be given in the paper. For the time being it is only interesting to observe how the system (2) is not a (reduced-order) observer of the state $x$ of (1) whose design, indeed, is not trivial due to the presence of the uncertain parameter $w$. Thus, the proposed design procedure can not be classified as "observed-based". Remarkably the previous results can be generalized to handle a wider class of systems and stabilization problems as detailed in the next sections.

\section{B. Framework and the Main Result}

The main goal of this paper is to present a design tool to handle the presence of asymptotically but not necessarily exponentially stable zero dynamics in robust output-feedback stabilization problems of nonlinear systems. Although the tool we are going to present lends itself to be useful in a significant variety of control scenarios, in order to keep confined the discussion while maintaining a certain degree of generality, we focus our attention on the class of smooth systems of the form

$$
\begin{aligned}
& \dot{x}=f(w, x, y) \quad x \in \mathbb{R}^{n}, \quad n \geq 0 \\
& \dot{y}=\kappa A y+B(q(w, x, y)+v) \quad y \in \mathbb{R}^{r}, \quad r \geq 1
\end{aligned}
$$

with measurable output

$$
y_{m}=C y \quad y_{m} \in \mathbb{R}
$$

in which the linear system $(A, B, C)$ is assumed to have relative degree $r$ with the pair $(A, C)$ observable with $A$ Hurwitz, $\kappa$ is a positive design parameter and $v$ is a control input. In the previous system the variable $w \in \mathbb{R}^{s}$ represents an exogenous variable which is governed by

$$
\dot{w}=s(w) \quad w \in W \subset \mathbb{R}^{s}
$$

with $W$ a compact set which is invariant for (4). As a particular case, the signals $w(t)$ generated by (4) may be constant, i.e. $s(w) \equiv 0$, namely constant uncertain parameters taking value in the set $W$ and affecting the system (3). In general, the variables $w$ can be considered as exogenous signals which, depending on the considered control scenario, may represent references to be tracked and/or disturbances to be rejected.

Remark 1: As a consequence of the fact that $W$ is a (forward and backward) invariant set for (4), the closed cylinder $\mathcal{C}_{n+r}:=W \times \mathbb{R}^{n+r}$ is invariant for (3), (4). Thus it is natural to regard system (3), (4) as a system evolving on $\mathcal{C}_{n+r}$ and endow the latter with the relative topology. This will be done from now on by referring to system (3), (4). Analogously, the dynamics described by the first $n$ equations of (3) and by (4) will be thought as evolving on the closed set $\mathcal{C}_{n}:=W \times \mathbb{R}^{n}$ which will be endowed with the relative topology. $\triangleleft$

We shall study the previous system under the following "minimum-phase" assumption.

Assumption: There exists a compact set $\mathcal{A} \subset \mathcal{C}_{n}$ which is locally asymptotically stable for the system

$$
\begin{aligned}
\dot{w} & =s(w) \\
\dot{x} & =f(w, x, 0) . \triangleleft
\end{aligned}
$$

Under this assumption, there exists a compact set $\mathcal{X} \subset \mathcal{C}_{n}$ such that $\mathcal{A} \subset \operatorname{int} \mathcal{X}$ and $\mathcal{A}$ is $\operatorname{LAS}(\mathcal{X})$ for system (5).

In this framework we consider the output feedback stabilization problem which consists of designing a locally Lipschitz regulator of the form

$$
\dot{\eta}=\varphi_{k}\left(\eta, y_{\mathrm{m}}\right) \quad v=\rho_{k}\left(\eta, y_{\mathrm{m}}\right) \quad \eta \in \mathbb{R}^{\nu}
$$

and, given arbitrary bounded sets $\mathcal{Y} \subset \mathbb{R}^{r}$ and $\mathcal{N} \subset \mathbb{R}^{\nu}$, a positive $\kappa^{\star}$, such that for all $\kappa \geq \kappa^{\star}$ and for some $\mathcal{B} \subset \mathbb{R}^{\nu+n}$ the set $\mathcal{B} \times\{0\}$ is $\operatorname{LAS}(\mathcal{N} \times \mathcal{X} \times \mathcal{Y})$ for the closed-loop system (3), (6).

The important point here is that $\varphi_{k}$ and $\rho_{k}$ must be locally Lipschitz. This restriction has strong practical motivations like sensitivity to noise or numeric and discrete time implementation.

Furthermore, we observe that the presence of the exogenous signal $w$, which is not available for feedback, makes the previous control framework structurally uncertain and requires the development of robust control laws.

The goal of the following part is to present a result regarding the solution of the robust stabilization problem formulated above. In order to ease the notation, in the following we shall drop in (3) the dependence from the variable $w$ which, in turn, will be thought as embedded in the variable $x$ (with the latter varying in the set $\mathcal{C}_{n}$ ). This, with a mild abuse of notation, will allow us to rewrite system (3) and (4) in the more compact form

$$
\begin{aligned}
\dot{x} & =f(x, y) \quad x \in \mathcal{C}_{n} \subset \mathbb{R}^{s+n} \\
\dot{y} & =\kappa A y+B(q(x, y)+v) \quad y \in \mathbb{R}^{r} \\
y_{m} & =C y
\end{aligned}
$$


and system (5) as $\dot{x}=f(x, 0)$.

The existence of a locally Lipschitz regulator solving the problem at hand, will be claimed under an assumption which involves the ability of asymptotically reproducing the function $q(x(t), 0)$, where $x(t)$ is any solution of $\dot{x}=f(x, 0)$ which can be generated by taking initial conditions on $\mathcal{A}$, by means of a locally Lipschitz system properly defined. The following definition aims to formally state the required reproducibility conditions which will be then used in the forthcoming Theorem 2.

Definition 1: (LER, ISS-LER): A triplet $(F(\cdot), Q(\cdot), \mathcal{A})$, where $F: \mathbb{R}^{m} \rightarrow \mathbb{R}^{m}$ and $Q: \mathbb{R}^{m} \rightarrow \mathbb{R}$ are smooth functions and $\mathcal{A} \subset \mathbb{R}^{m}$ is a compact attractor for $\dot{z}=F(z)$, is said to be Locally Exponentially Reproducible (LER) if there exists a compact set $\mathcal{R} \supseteq \mathcal{A}$ contained in the domain of attraction of $\mathcal{A}$ which is LES for $\dot{z}=F(z)$ and, for any bounded set $\mathcal{Z}$ contained in the domain of attraction of $\mathcal{R}$, there exist an integer $p$, locally Lipschitz functions $\varphi: \mathbb{R}^{p} \rightarrow \mathbb{R}^{p}$, $\gamma: \mathbb{R}^{p} \rightarrow \mathbb{R}$, and $\psi: \mathbb{R}^{p} \rightarrow \mathbb{R}^{p}$, with $\psi$ a complete vector field, and a locally Lipschitz function $T: \mathbb{R}^{m} \rightarrow \mathbb{R}^{p}$, such that

$$
Q(z)+\gamma(T(z))=0 \quad \forall z \in \mathcal{R}
$$

and for all $\xi_{0} \in \mathbb{R}^{p}$ and $z_{0} \in \mathcal{Z}$ the solution $(\xi(t), z(t))$ of

$$
\begin{aligned}
& \dot{z}=F(z) \quad z(0)=z_{0} \\
& \dot{\xi}=\varphi(\xi)+\psi(\xi) Q(z) \quad \xi(0)=\xi_{0}
\end{aligned}
$$

satisfies

$$
|(\xi(t), z(t))|_{\left.\operatorname{graph} T\right|_{\mathcal{R}}} \leq \beta\left(t,\left|\left(\xi_{0}, z_{0}\right)\right|_{\left.\operatorname{graph} T\right|_{\mathcal{R}}}\right)
$$

where $\beta(\cdot, \cdot)$ is a locally exponentially class- $\mathcal{K} \mathcal{L}$ function.

Furthermore the triplet in question is said to be Input-to-State Locally Exponentially Reproducible (ISS-LER) if it is LER and, in addition, for all locally essentially bounded $v(t)$, for all $\xi_{0} \in$ $\mathbb{R}^{p}$ and $z_{0} \in \mathcal{Z}$ the solution $(\xi(t), z(t))$ of

$$
\begin{aligned}
& \dot{z}=F(z) \quad z(0)=z_{0} \\
& \dot{\xi}=\varphi(\xi)+\psi(\xi)[Q(z)+v(t)] \quad \xi(0)=\xi_{0}
\end{aligned}
$$

satisfies

$$
\begin{aligned}
|(\xi(t), z(t))|_{\left.\operatorname{graph} T\right|_{\mathcal{R}}} \leq \beta\left(t, \mid\left(\xi_{0}, z_{0}\right)\right. & \left.\left.\right|_{\left.\operatorname{graph} T\right|_{\mathcal{R}}}\right) \\
& +\ell\left(\sup _{\tau \leq t}|v(\tau)|\right)
\end{aligned}
$$

where $\beta(\cdot, \cdot)$ is a locally exponentially class- $\mathcal{K} \mathcal{L}$ function and $\ell$ is a class- $\mathcal{K}$ function. $\triangleleft$

We postpone to Section II-C a broad discussion about this definition and to Section IV the presentation of sufficient conditions for a triplet to be ISS-LER.

With this definition at hand, we pass to formulate the following theorem which fixes a framework where the stabilization problem previously formulated can be solved by means of a locally Lipschitz regulator.

Theorem 2: Let $\mathcal{A}$ be $\operatorname{LAS}(\mathcal{X})$ for the system $\dot{x}=f(x, 0)$ for some compact set $\mathcal{X} \subset \mathcal{C}_{n}$. Assume, in addition, that the triplet $(f(x, 0), q(x, 0), \mathcal{A})$ is $L E R$. Then there exist a locally Lipschitz regulator of the form (6), a compact set $\mathcal{R} \supseteq \mathcal{A}$, a continuous function $\tau: \mathcal{R} \rightarrow \mathbb{R}^{\nu}$, and, for any compact set $\mathcal{Y} \subset \mathbb{R}^{r}$ and $\mathcal{N} \subset \mathbb{R}^{\nu}$, a positive constant $\kappa^{\star}$, such that for all $\kappa \geq \kappa^{\star}$ the set

$\operatorname{graph} \tau \times\{0\}=\left\{(\eta, x, y) \in \mathbb{R}^{\nu} \times \mathcal{R} \times \mathbb{R}^{r}: \eta=\tau(x), y=0\right\}$

is $\operatorname{LES}(\mathcal{N} \times \mathcal{X} \times \mathcal{Y})$ for (7), (6) and the set

$\left.\operatorname{graph} \tau\right|_{\mathcal{A}} \times\{0\}=\left\{(\eta, x, y) \in \mathbb{R}^{\nu} \times \mathcal{A} \times \mathbb{R}^{r}: \eta=\tau(x), y=0\right\}$

is $\operatorname{LAS}(\mathcal{N} \times \mathcal{X} \times \mathcal{Y})$ for (7), (6). Furthermore, if $\mathcal{A}$ is also LES for the system $\dot{x}=f(x, 0)$, the set $\mathcal{R}$ can be taken equal to $\mathcal{A}$.

Proof: By the definition of LER of the triplet $(f(x, 0), q(x, 0), \mathcal{A})$ there exists a set $\mathcal{R} \supseteq \mathcal{A}$ which is LES for $\dot{x}=f(x, 0)$ and, for any compact set $\mathcal{X}_{1} \subset \mathcal{D}(\mathcal{R})$, there exist an integer $\nu$, locally Lipschitz functions $\varphi: \mathbb{R}^{\nu} \rightarrow \mathbb{R}^{\nu}$, $\psi: \mathbb{R}^{\nu} \rightarrow \mathbb{R}^{\nu}, \gamma: \mathbb{R}^{\nu} \rightarrow \mathbb{R}$ and a locally Lipschitz function $T: \mathbb{R}^{n} \rightarrow \mathbb{R}^{\nu}$ such that

$$
q(x, 0)+\gamma(T(x))=0 \quad \forall x \in \mathcal{R}
$$

and for all $\xi_{0} \in \mathbb{R}^{p}$ and $x_{0} \in \mathcal{X}_{1}$ the solution $(\xi(t), x(t))$ of

$$
\begin{aligned}
\dot{x} & =f(x, 0) \quad x(0)=x_{0} \\
\dot{\xi} & =\varphi(\xi)+\psi(\xi) q(x, 0) \quad \xi(0)=\xi_{0}
\end{aligned}
$$

satisfies

$$
|(\xi(t), x(t))|_{\left.\operatorname{graph} T\right|_{\mathcal{R}}} \leq \beta\left(t,\left|\left(\xi_{0}, x_{0}\right)\right|_{\left.\operatorname{graph} T\right|_{\mathcal{R}}}\right)
$$

where $\beta(\cdot, \cdot)$ is a locally exponentially class- $\mathcal{K} \mathcal{L}$ function. As $\mathcal{A}$ is $\operatorname{LAS}(\mathcal{X})$ and $\mathcal{R} \supseteq \mathcal{A}$ and contained in the domain of attraction of $\mathcal{A}$, we have $\mathcal{D}(\mathcal{R})=\mathcal{D}(\mathcal{A})$ and thus the previous properties hold, in particular, with $\mathcal{X}_{1}=\mathcal{X}$. Furthermore, in case where $\mathcal{A}$ is LES for $\dot{x}=f(x, 0)$, it is possible to show that (15) and (17) hold also with $\mathcal{R}$ replaced by $\mathcal{A}$ possibly with a different class- $\mathcal{K} \mathcal{L}$ function $\beta(\cdot, \cdot)$.

Assume, without loss of generality (as $(A, B, C)$ has relative degree $r$ and $(A, C)$ is observable), that the pair $(A, C)$ is in the canonical observability form and that $B=(0, \ldots, 0,1)^{\mathrm{T}}$, and choose, as candidate controller, the system

$$
\begin{aligned}
& \dot{\eta}=\varphi(\eta)-\psi(\eta) \gamma(\eta)-\psi(\eta) \kappa B^{\mathrm{T}} A y \\
& v=\gamma(\eta)
\end{aligned}
$$

which, by the structure of $A$ and $B$, is of the form (6) since $B^{T} A y=a_{r} y_{m}$ where $a_{r}$ is the element of $A$ in the $r$ th row and first column.

Consider now the change of variables

$$
\eta \rightarrow \chi:=\phi_{\psi}\left(B^{\mathrm{T}} y, \eta\right)
$$

Note that such a change of variables is well-defined for all $y$ and $\eta$ as $\psi$ is complete.

Since

$$
\frac{\partial \phi_{\psi}\left(t^{\prime}, \eta\right)}{\partial t^{\prime}}-\frac{\partial \phi_{\psi}\left(t^{\prime}, \eta\right)}{\partial \eta} \psi(\eta) \equiv 0
$$


and using the fact that

$$
\left.\frac{\partial \phi_{\psi}\left(t^{\prime}, \eta\right)}{\partial t^{\prime}}\right|_{t^{\prime}=0}=\left.\psi(\eta) \quad \frac{\partial \phi_{\psi}\left(t^{\prime}, \eta\right)}{\partial \eta}\right|_{t^{\prime}=0}=I
$$

it turns out that the closed-loop dynamics (3), (18) in the new coordinates can be described as the feedback interconnection of a system of the form

$$
\begin{aligned}
& \dot{x}=f(x, 0)+\tilde{f}(x, y) \\
& \dot{\chi}=\varphi(\chi)+\psi(\chi) q(x, 0)+\tilde{\ell}_{1}(x, \chi, y)
\end{aligned}
$$

and a system of the form

$$
\dot{y}=\kappa A y+B(q(x, 0)+\gamma(\chi))+\tilde{\ell}_{2}(x, \chi, y)
$$

in which $\tilde{f}(x, y), \tilde{\ell}_{1}(x, \chi, y)$ and $\tilde{\ell}_{2}(x, \chi, y)$ are locally Lipschitz functions satisfying $\tilde{f}(x, 0)=0, \tilde{\ell}_{1}(x, \chi, 0)=0$ and $\tilde{\ell}_{2}(x, \chi, 0)=0$ for all $x \in \mathcal{C}$ and $\chi \in \mathbb{R}^{\nu}$ and with $\tilde{\ell}_{1}$ and $\tilde{\ell}_{2}$ possibly dependent on $\mathcal{X}$.

Let $\mathcal{N}$ and $\mathcal{Y}$ be arbitrary compact sets of $\mathbb{R}^{\nu}$ and $\mathbb{R}^{r}$ and denote by $\Xi$ the compact set in which $\chi$ ranges if $\eta$ and $y$ range within $\mathcal{N}$ and $\mathcal{Y}$, respectively (note that $\mathcal{N}$ may depend on $\mathcal{X}$ via the function $\psi$ ). Since system (20) with $y=0$ is nothing but (16), it turns out that graph $\left.T\right|_{\mathcal{R}}$ is $\operatorname{LES}(\mathcal{X} \times \Xi)$ for system (20) with $y=0$. Furthermore, by (15), the term $q(x, 0)+\gamma(\chi)$ in (21) is identically zero for $\left.(x, \chi) \in \operatorname{graph} T\right|_{\mathcal{R}}$. From these facts, the fact that $A$ is Hurwitz, and the results in [1], [28], it follows that for any compact set $\mathcal{Y} \in \mathbb{R}^{r}$ there exists a $\kappa^{\star}>0$ such that for all $\kappa \geq \kappa^{\star}$ the set graph $\left.T\right|_{\mathcal{R}} \times\{0\}$ is $\operatorname{LES}(\mathcal{X} \times \Xi \times \mathcal{Y})$ for (20), (21). By taking $\tau=\left.T\right|_{\mathcal{R}}$ the previous result proves the first part of the theorem, namely that graph $\tau \times\{0\}$ is $\operatorname{LES}(\mathcal{X} \times \mathcal{Y} \times \mathcal{N})$ for the closed-loop system (3), (18).

We prove now the second claim of the theorem, namely that graph $\left.\tau\right|_{\mathcal{A}} \times\{0\}$ is $\operatorname{LAS}(\mathcal{X} \times \mathcal{Y} \times \mathcal{N})$. Let $\kappa \geq \kappa^{\star}$ be fixed and note that, as graph $\tau \times\{0\}$ attracts uniformly the closed-loop trajectories leaving $\mathcal{X} \times \mathcal{Y} \times \mathcal{N}$, Proposition 4 in Appendix yields that

$$
\omega(\mathcal{X} \times \mathcal{Y} \times \mathcal{N})=\omega(\operatorname{graph} \tau \times\{0\}) \subseteq \operatorname{graph} \tau \times\{0\}
$$

in which $\omega(S)$ denotes the omega limit set of the set $S$ associated to the closed-loop system. We prove now that if $(x, y, \chi) \in$ $\omega($ graph $\tau \times\{0\})$ then necessarily $x \in \omega(\mathcal{R})$ in which $\omega(\mathcal{R})$ denotes the omega limit set of the set $\mathcal{R}$ associated to the system $\dot{x}=f(x, 0)$. Indeed, consider a sequence $\left\{x_{n}, y_{n}, \chi_{n}\right\}$ with $\left(x_{n}, \chi_{n}\right) \in \operatorname{graph} \tau$ and so in particular $x_{n} \in \mathcal{R}$, and $y_{n} \equiv 0$, and a divergent sequence $\left\{t_{n}\right\}$, such that the following holds:

$$
\left|x\left(t_{n}, x_{n}\right)-\bar{x}\right| \rightarrow 0
$$

where $x\left(t, x_{n}\right)$ and $\chi\left(\left(x_{n}, \chi_{n}\right), t\right)$ denotes the solution of

$$
\begin{aligned}
& \dot{x}=f(x, 0) \\
& \dot{\chi}=\varphi(\chi)+\psi(\chi) q(x, 0)
\end{aligned}
$$

with initial conditions $\left(x_{n}, \chi_{n}\right) . x_{n}$ being in $\mathcal{R}$, this implies $\bar{x} \in \omega(\mathcal{R})$. Now, considering the system given by the first dynamics in (23) and using the fact that $\mathcal{A} \subseteq \mathcal{R}$ uniform attracts the trajectories of this system leaving $\mathcal{X}$, Proposition 4 in Appendix yields that $\omega(\mathcal{X})=\omega(\mathcal{R})=\omega(\mathcal{A}) \subseteq \mathcal{A}$. By this and the previous arguments we conclude that the $x$ components of the closed-loop trajectories are uniformly attracted by $\omega(\mathcal{A}) \subseteq \mathcal{A}$. From this the result follows by standard arguments.

Remark 3: By going throughout the proof of the previous theorem, it turns out that the regulator (6) solving the problem at hand has the form

$$
\begin{aligned}
& \dot{\eta}=\varphi(\eta)-\psi(\eta)\left[\gamma(\eta)+\kappa B^{\mathrm{T}} A y\right] \\
& v=\gamma(\eta)
\end{aligned}
$$

with $\kappa$ the sufficiently large positive number introduced in (3) and $(\varphi(\cdot), \psi(\cdot), \gamma(\cdot))$ the locally Lipschitz functions which are associated to the triplet $(f(x, 0), q(x, 0), \mathcal{A})$ in the definition of local exponential reproducibility. $\triangleleft$

\section{A Brief Digression About the Problem}

The structure of (3) and the associated problem, apparently very specific, are indeed recurrent in a number of control scenarios in which robust non linear stabilization is involved. We refer to Section III-A for the presentation of a few relevant cases where this occurs.

The rich available literature on nonlinear stabilization already provides successful tools to solve the problem at hand if the assumption above is strengthen by asking that the set $\mathcal{A}$ is also $\operatorname{LES}(\mathcal{X})$ for $(5)$. As a matter of fact, under the previous condition, it is a well-known fact that a large value of $\kappa$, with $v \equiv 0$, suffices to solve the problem as formalized in ([1], [28]). In the case $\mathcal{A}$ is not LES for (5) the only conclusion which can be drawn if $v \equiv 0$ is that the origin is semiglobally practically stable in the parameter $\kappa$, that is the trajectories of the system can be steered arbitrary close to the set $\mathcal{A} \times\{0\}$ by increasing the value of $\kappa$ (see [1], [7], [22], [28]). Indeed, the simple example discussed in Section II-A gives evidence of the previous fact. In these critical scenarios an appropriate design of the control input $v$ becomes inevitable in order to compensate for the interconnection term $q(w, x, y)$ between output and zero dynamics which cannot be simple dominated by linear high gain.

In particular, a first possible option, motivated by small gain arguments and gain assignment procedures for nonlinear systems (see [16], [17], [30]), is to design the control $v$ in order to assign, to the $y$-subsystem, a certain nonlinear ISS gain suitably identified according to small gain criterions and to the asymptotic gain of the $x$-subsystem (3). This option, however, necessarily leads to design control laws which are not, in general, locally Lipschitz close to the compact attractor and, thus, which violate a basic requirement of the above problem.

An alternative option to design the control $v$ is to be inspired by nonlinear separation principles (see, besides others, [1], [11], [15], [28], [29]), namely to design an appropriate state observer yielding an asymptotic estimate $(\hat{w}, \hat{x}, \hat{y})$ of the state variables, and to asymptotically compensate for the coupling term $q(w, x, y)$ by implementing a "certainty equivalence" control law of the form $v=-q(\hat{w}, \hat{x}, \hat{y})$. Indeed, under suitable conditions, the tools proposed in [29] would allow one to precisely fix the details and to solve the problem at hand in a rigorous way. This way of approaching the problem, though, presents a number of drawbacks which substantially limit its 
applicability. First, the design of the observer clearly requires the formulation of suitable observability assumptions ${ }^{1}$ on the controlled plant, and in particular of its $(w, x)$ components, not in principle necessary for the stabilization problem to be solvable, which may be not fulfilled for a number of relevant cases. Furthermore, it is worth noting how approaching the problem according to the previous design philosophy, leads to inherently redundant control structures, by requiring the explicit estimate of the full state (and of possible uncertainties) in order to reproduce the signal $q(w, x, y)$.

As opposite to the previous strategies (and according to the principles explained in Section II-A), Theorem 2 provides a design procedure based on asymptotic reconstruction of the interconnection term $q(w, x, y)$, not relying upon the design of an observer of the state variables $(w, x, y)$. In this respect the crucial property underlying the Theorem is the local exponential reproducibility property which, according to its definition, relies upon two requirements (whose importance in the solution of the problem can be better understood having in mind the example and the discussion in Section II-A). The key first requirement, for a triplet $(F, Q, \mathcal{A})$ to be LER, is that there exists a set $\mathcal{R}$ which contains $\mathcal{A}$ and which is LES for the autonomous system $\dot{z}=F(z)$. As anticipated in Section II-A, where the intuition about the role of the set $\mathcal{R}$ in the stabilization problem has been given, the existence of the set $\mathcal{R}$ is always guaranteed if the set $\mathcal{A}$ is LAS for $\dot{z}=F(z)$ (see the forthcoming Lemma 1). Thus, put in the perspective of Theorem 2, the first property of LER is always fulfilled.

The second crucial requirement characterizing the definition is that there exists a locally Lipschitz system of the form

$$
\begin{aligned}
\dot{\xi} & =\varphi(\xi)+\psi(\xi) u_{\xi} \\
y_{\xi} & =\gamma(\xi)
\end{aligned}
$$

with input $u_{\xi}$ and output $y_{\xi}$, such that system (9), modeling the cascade connection of the autonomous system $\dot{z}=F(z)$ with output $y_{z}=Q(z)$ with the system (24), has a locally exponentially stable set described by graph $\left.T\right|_{\mathcal{R}}$ and, on this set, the output $y_{\xi}$ equals $y_{z}$ (see (8)). The domain of attraction of graph $\left.T\right|_{\mathcal{R}}$ is required to be of the form $\mathcal{Z} \times \mathbb{R}^{p}$ with $\mathcal{Z}$ any compact set in the domain of attraction of $\mathcal{R}$ (note that, according to the definition, system (24) is allowed to depend on the choice of $\mathcal{Z}$ ). In this respect the second requirement can be regarded as the ability, of the system (24), of asymptotically reproducing the output function $Q(z(t))$ of system $\dot{z}(t)=F(z(t))$ with initial conditions of the latter taken in $\mathcal{Z}$. By bearing in mind the discussion in Section II-A and the statement of Theorem 2 (in which the LER notion is applied to the triplet $(f(x, 0), q(x, 0), \mathcal{A}))$, the above conditions precisely fix the technicalities needed to design a stabilizer embedding an "internal model" of the zero dynamics closed to the asymptotic attractor $\mathcal{A}$ and thus capable to offset the coupling term $q(x(t), 0)$ for any $x(t)$ evolving on $\mathcal{R}$.

Remark 4: Note how the "output reproducibility" property required to system (24) does not hide, in principle, any kind of

\footnotetext{
${ }^{1}$ It must be noted that only local observability notion are potentially needed at this level as a consequence of the fact that practical stability is already guaranteed by the high-gain law $\kappa$.
}

state observability property of the system $\dot{z}=F(z)$ with output $y_{z}=Q(z)$. In other words system (24) must not be confused with a state observer of the $z$-subsystem as its role is to estimate only its output $Q(z)$ and not its entire state $z$.

As the definition of $I S S$-LER, we only note that, in addition to the previous properties, it is required that system (11) exhibits an ISS property (without any special requirement on the asymptotic gain) with respect to the exogenous input $v$.

\section{APPLICATIONS}

\section{A. Output-Feedback From UCO State-Feedback in Presence of Non-Hyperbolic Attractors}

In this part we show how the theory of robust nonlinear separation principle presented in [1], [28] can be extended with the tools developed in the previous sections. In particular we are interested to extend the theory of [28] by showing how to design a pure output-feedback semiglobal controller stabilizing an attractor when it is known how the latter can be asymptotically (but not exponentially) stabilized by means of a Uniform Completely Observable (UCO) state-feedback controller.

Consider the system

$$
\begin{aligned}
\dot{w} & =s(w) \quad w \in W \subset \mathbb{R}^{s} \\
\dot{z} & =A(w, z, u) \quad z \in \mathbb{R}^{m}, u \in \mathbb{R} \\
y & =C(w, z) \quad y \in \mathbb{R}
\end{aligned}
$$

in which $u$ and $y$ are respectively the control input and the measured output and $W$ is a compact set which is invariant for $\dot{w}=s(w)$. As discussed in the previous section, the variables $w$ emphasize the possible presence of parametric uncertainties and/or disturbance to be rejected and/or reference to be tracked (in the latter case the measurable output $y$ plays more likely the role of regulation/tracking error). As done before, in order to simplify the notation, we drop the dependence of the variable $w$ and we compact system (25) in the more convenient form

$$
\begin{aligned}
& \dot{z}=A(z, u) \quad z \in \mathbb{R}^{m}, u \in \mathbb{R} \\
& y=C(z) \quad y \in \mathbb{R}
\end{aligned}
$$

which is supposed to evolve on a closed invariant set $\mathcal{C}_{m}$ which is endowed with the subset topology (such a closed set being, in the form (25), the closed cylinder $\mathcal{C}_{m}:=W \times \mathbb{R}^{m}$ ).

We recall (see [28]) that a function $\bar{u}: \mathbb{R}^{m} \rightarrow \mathbb{R}$ is said to be UCO with respect to (26) if there exist two integers $n_{y}, n_{u}$ and a $\mathcal{C}^{1}$ function $\Psi$ such that, for each solution of

$$
\begin{aligned}
\dot{z} & =A\left(z, u_{0}\right) \\
\dot{u}_{i} & =u_{i+1} \quad i=0, \ldots, n_{u}-1 \\
\dot{u}_{n_{u}} & =v
\end{aligned}
$$

we have, for all $t$ where the solution makes sense

$$
\bar{u}(z(t))=\Psi\left(y(t), y^{(1)}(t), \ldots, y^{\left(n_{y}\right)}(t), u_{0}(t), \ldots, u_{n_{u}}(t)\right)
$$

where $y^{(i)}(t)$ denotes the $i$ th derivative of $y$ at time $t$.

Motivated by [28] we shall study system (26) under the following two assumptions: 
a) there exist a smooth function $\bar{u}: \mathbb{R}^{m} \rightarrow \mathbb{R}$ and compact sets $\mathcal{A} \subset \mathcal{C}_{m}$ and $\mathcal{Z} \subset \mathcal{C}_{m}$, such that $\mathcal{A}$ is $\operatorname{LAS}(\mathcal{Z})$ for system (26) with $u=\bar{u}(z) ; 2$

b) $\bar{u}(z)$ is UCO with respect to (26).

In this framework we shall be able to prove, under suitable reproducibility conditions specified later, that the previous two assumptions imply the existence of a locally Lipschitz dynamic output feedback regulator able to asymptotically stabilize the set $\mathcal{A}$. The main theorem in this direction is detailed next. In this theorem we refer to an integer $\ell_{u}$ defined as that number such that for the system

$$
\begin{aligned}
\dot{z} & =A\left(z, \xi_{0}\right) \\
\dot{\xi}_{i} & =\xi_{i+1} \quad i=0, \ldots, \ell_{u}-1 \\
\dot{\xi}_{\ell_{u}} & =u_{1} \\
y & =C(z)
\end{aligned}
$$

there exist smooth functions $C_{i}$ such that the first $n_{y}+1$ time derivatives of $y$ can be expressed as

$$
y^{(i)}=C_{i}\left(z, \xi_{0}, \ldots, \xi_{\ell_{u}}\right) \quad \forall i=0, \ldots, n_{y}+1 .
$$

Without loss of generality we assume $\ell_{u} \geq n_{u}$.

Theorem 5: Consider system (26) and assume the existence of a compact set $\mathcal{A} \subset \mathcal{C}_{m}$ and of a smooth function $\bar{u}(z)$ such that properties (a) and (b) specified above are satisfied. Assume, in addition, that the triplets

$$
\left(A(z, \bar{u}(z)), L_{A(z, \bar{u}(z))}^{\left(\ell_{u}+1\right)} \bar{u}(z), \mathcal{A}\right)
$$

and

$$
\left(A(z, \bar{u}(z)), L_{A(z, \bar{u}(z))}^{\left(n_{y}+1\right)} C(z), \mathcal{A}\right)
$$

are ISS-LER. Then there exist a positive $o$, a compact set $\mathcal{B} \subset$ $\mathbb{R}^{o}$ and, for any $\mathcal{N} \subset \mathbb{R}^{o}$, a locally Lipschitz controller of the form

$$
\begin{aligned}
& \dot{\zeta}=\Phi(\zeta, y) \quad \zeta \in \mathbb{R}^{o} \\
& u=\Upsilon(\zeta, y)
\end{aligned}
$$

such that the set $\mathcal{A} \times \mathcal{B}$ is $\operatorname{LAS}(\mathcal{Z} \times \mathcal{N})$ for the closed-loop system (26), (32).

This result extends Theorem 1.1 of [28] in three directions. First, note that we are dealing with stabilization of compact attractors for systems evolving on closed sets. This is a technical improvement on which, though, we would not like to put the emphasis. Second, note that the UCO control law $\bar{u}(z)$ is not required to be vanishing on the attractor $\mathcal{A}$ which, as a consequence, is not required to be forward invariant for the open loop system (26) with $u \equiv 0$. In this respect the proposed setting can be seen as also able to frame output regulation problems.

${ }^{2} \mathrm{By}$ referring to (25), a meaningful case to be considered is when $\mathcal{A}=$ $W \times\{0\}$, in which case this assumption amounts to require the existence of a state feedback stabilizer, possibly dependent on the uncertainties, able to asymptotically stabilize the origin with a certain domain of attraction.
Finally, the previous result claims that, by means of a pure $l o$ cally Lipschitz output feedback controller, we are able to restore the asymptotic properties of an UCO controller without relying upon exponential stability requirements of the latter and with the ability to cope with uncertain parameters included in the model. The last two extensions are conceptually very much relevant and can be seen as particular applications of the tools presented in the previous sections.

Following the main laying of [28], the proof of Theorem 5 immediately follows by combining the analysis contained in the following two subsections which contain results interesting on their own.

\section{B. Robust Asymptotic Backstepping}

In this part we discuss how the UCO control law $\bar{u}$ can be robustly back-step through the chain of integrators of (27). As commented above, the forthcoming proposition extends in a not trivial way the results of [28] in the measure in which one considers the fact that $\bar{u}(z)$ is not vanishing on the attractor and that $\mathcal{A}$ is not necessarily locally exponential stable for the closed-loop system.

We show that the existence of the static UCO stabilizer for (26) implies the existence of a dynamic stabilizer for (29) using the partial state $\xi_{i}, i=0, \ldots, \ell_{u}$, and the output derivatives $y^{(i)}$, $i=1, \ldots, n_{y}$. This is formally proved in the next proposition.

Proposition 1: Consider system (29) under the assumption (a) previously formulated. Assume that the triplet (30) is ISSLER. Then there exists a positive $\nu$, a compact set $\mathcal{R} \supset \mathcal{A}$, a continuous function $\tau: \mathcal{R} \rightarrow \mathbb{R}^{\nu+\ell_{u}+1}$, and, for any compact set $\Xi^{\prime} \subset \mathbb{R}^{\ell_{u}+1}$ and $\mathcal{N}^{\prime} \subset \mathbb{R}^{\nu}$, a locally Lipschitz regulator of the form

$$
\begin{aligned}
\dot{\eta} & =\varphi(\eta, \xi, \bar{u}(z)) \quad \eta \in \mathbb{R}^{\nu} \\
u_{1} & =\rho(\eta, \xi, \bar{u}(z))
\end{aligned}
$$

with $\xi=\operatorname{col}\left(\xi_{0}, \ldots, \xi_{\ell_{u}}\right)$ such that the sets

$$
\operatorname{graph} \tau=\left\{(z, \xi, \eta) \in \mathcal{R} \times \mathbb{R}^{\ell_{u}+1} \times \mathbb{R}^{\nu}:(\xi, \eta)=\tau(z)\right\}
$$

and

$\left.\operatorname{graph} \tau\right|_{\mathcal{A}}:=\left\{(z, \xi, \eta) \in \mathcal{A} \times \mathbb{R}^{\ell_{u}+1} \times \mathbb{R}^{\nu}:(\xi, \eta)=\tau(z)\right\}$

are, respectively, $\operatorname{LES}\left(\mathcal{Z} \times \Xi^{\prime} \times \mathcal{N}^{\prime}\right)$ and $\operatorname{LAS}\left(\mathcal{Z} \times \Xi^{\prime} \times \mathcal{N}^{\prime}\right)$ for the closed-loop system (29), (33).

Proof: Consider the change of variables

$$
\begin{aligned}
\xi_{0} & \rightarrow \tilde{\xi}_{0}:=\xi_{0}-\bar{u}(z) \\
\xi_{i} & \rightarrow \tilde{\xi}_{i}:=\xi_{i}-\bar{u}^{(i)}\left(z, \tilde{\xi}_{0}, \ldots, \tilde{\xi}_{i-1}\right) \quad i=1, \ldots, \ell_{u}
\end{aligned}
$$

where the $\bar{u}^{(i)}(z), i=1, \ldots, \ell_{u}$, are recursively defined as

$$
\begin{aligned}
\bar{u}^{(1)}\left(z, \tilde{\xi}_{0}\right):= & \frac{\partial \bar{u}(z)}{\partial z} A\left(z, \tilde{\xi}_{0}+\bar{u}(z)\right) \\
\bar{u}^{(i)}:= & \frac{\partial \bar{u}^{(i-1)}\left(z, \tilde{\xi}_{0}, \ldots \tilde{\xi}_{i-2}\right)}{\partial z} A\left(z, \tilde{\xi}_{0}+\bar{u}(z)\right) \\
& +\sum_{j=0}^{i-2} \frac{\partial \bar{u}^{(i-1)}\left(z, \tilde{\xi}_{0}, \ldots \tilde{\xi}_{i-2}\right)}{\partial \tilde{\xi}_{j}} \tilde{\xi}_{j+1}
\end{aligned}
$$


for $i=2, \ldots, \ell_{u}$, where $\bar{u}^{(i)}=\bar{u}^{(i)}\left(z, \tilde{\xi}_{0}, \ldots, \tilde{\xi}_{i-1}\right)$ and the further change of variable

$$
\begin{aligned}
\tilde{\xi}_{i} \rightarrow \zeta_{i} & :=g^{-i} \tilde{\xi}_{i} \quad i=0, \ldots, \ell_{u}-1 \\
\tilde{\xi}_{\ell_{u}} \rightarrow \zeta_{\ell_{u}} & :=\tilde{\xi}_{\ell_{u}}-\sum_{i=0}^{\ell_{u}-1} a_{i} g^{\ell_{u}-i} \tilde{\xi}_{i}
\end{aligned}
$$

where $g$ is a positive design parameter and the $a_{i}$ 's are coefficients of an Hurwitz polynomial.

By letting $\zeta:=\operatorname{col}\left(\zeta_{0}, \ldots, \zeta_{\ell_{u}-1}\right)$ system (29) in the new coordinates reads as

$$
\begin{aligned}
\dot{z} & =A(z, \bar{u}(z))+\tilde{A}(z, C \zeta) \\
\dot{\zeta} & =g H \zeta+B \zeta_{\ell_{u}} \\
\dot{\zeta}_{\ell_{u}} & =u_{1}+\ell_{g}\left(z, \zeta, \zeta_{\ell_{u}}\right)
\end{aligned}
$$

where $B=\operatorname{col}(0, \ldots, 0,1), C=(1,0, \ldots, 0), \tilde{A}(z, C \zeta)=$ $A\left(z, \tilde{\xi}_{0}+\bar{u}(z)\right)-A(z, \bar{u}(z)), H$ is a Hurwitz matrix and $\ell_{g}(\cdot)$ is a smooth function such that

$$
\ell_{g}(z, 0,0)=-L_{A(z, \bar{u}(z))}^{\left(\ell_{u}+1\right)} \bar{u}(z) \quad \forall z \in \mathbb{R}^{n} .
$$

As the triplet (30) is ISS-LER, there exists a compact set $\mathcal{R} \supseteq \mathcal{A}$ which is LES for $\dot{z}=A(z, \bar{u}(z))$ with $\mathcal{D}(\mathcal{R}) \supseteq \mathcal{D}(\mathcal{A})$. Furthermore, by the fact that (30) is ISS-LER and by definition of ISS-LER, the triplet $\left(A(z, \bar{u}(z)),-L_{A(z, \bar{u}(z))}^{\left(\ell_{u}+1\right)} \bar{u}(z), \mathcal{R}\right)$ is also ISS-LER. We consider now the zero dynamics, with respect to the input $u_{1}$ and output $\zeta_{\ell_{u}}$, of system (35) given by

$$
\begin{aligned}
& \dot{z}=A(z, \bar{u}(z))+\tilde{A}(z, C \zeta) \\
& \dot{\zeta}=g H \zeta .
\end{aligned}
$$

For this system it can be proved (by means of arguments which, for instance, can be found in [22], [28]) that for any compact set $\mathcal{M} \in \mathbb{R}^{\ell_{u}}$ there exists a $g^{\star}>0$ such that for all $g \geq g^{\star}$ the sets $\mathcal{R} \times\{0\}$ and $\mathcal{A} \times\{0\}$ are respectively $\operatorname{LES}(\mathcal{Z} \times \mathcal{M})$ and $\operatorname{LAS}(\mathcal{Z} \times \mathcal{M})$ for (37). Fix, once for all, $g \geq g^{\star}$. By the previous facts, by (36), by the fact that the triplet $\left(A(z, \bar{u}(z)),-L_{A(z, \bar{u}(z))}^{\left(\ell_{u}+1\right)} \bar{u}(z), \mathcal{R}\right)$ is ISS-LER, and by Proposition 5 in Appendix A, it follows that the triplet $\left((37), \ell_{g}(z, \zeta, 0), \mathcal{R} \times\{0\}\right)$ is LER. Now fix

$$
u_{1}=-\kappa \zeta_{\ell_{u}}+v
$$

where $\kappa$ is a positive design parameter and $v$ is a residual control input. From the previous results, it follows that system (35) with (38) fits into the framework of Theorem 2, by which it is possible to conclude that there exists a locally Lipschitz controller of the form

$$
\dot{\xi}=\Phi_{k}^{\prime}\left(\xi, \zeta_{\ell_{u}}\right) \quad v=\Upsilon_{k}^{\prime}\left(\xi, \zeta_{\ell_{u}}\right) \quad \xi \in \mathbb{R}^{p}
$$

a continuous function $\tau^{\prime}: \mathcal{R} \times\{0\} \rightarrow \mathbb{R}^{p}$ and, for any compact set $\mathcal{M}_{\ell_{u}} \subset \mathbb{R}$ and $\mathcal{N}^{\prime} \subset \mathbb{R}^{p}$, a positive constant $\kappa^{\star}$, such that for all $\kappa \geq \kappa^{\star}$ the set

$\operatorname{graph} \tau^{\prime} \times\{0\}=\left\{\left((z, \zeta), \zeta_{\ell_{u}}, \xi\right) \in(\mathcal{R} \times\{0\}) \times \mathbb{R} \times \mathbb{R}^{p}:\right.$

$$
\left.\zeta_{\ell_{u}}=0, \xi=\tau^{\prime}(z, \zeta)\right\}
$$

is $\operatorname{LES}\left(\mathcal{Z} \times \mathcal{M} \times \mathcal{M}_{\ell_{u}} \times \mathcal{N}^{\prime}\right)$ for (35), (38) and (39). Furthermore, by properly adapting the arguments at the end of the proof of Theorem 2, it is also possible to prove that the set graph $\left.\tau^{\prime}\right|_{\mathcal{A} \times\{0\}} \times\{0\}$ is $\operatorname{LAS}\left(\mathcal{Z} \times \mathcal{M} \times \mathcal{M}_{\ell_{u}} \times \mathcal{N}^{\prime}\right)$ for (35), (38) and (39).

The previous facts have shown how to solve the problem at hand by means of a regulator processing $\tilde{\xi}_{0}=\xi_{0}-\bar{u}(z)$ and its first $\ell_{u}$ time derivatives $\tilde{\xi}_{i}$ of the form

$$
\begin{aligned}
\dot{\xi} & =\Phi_{k}^{\prime}\left(\xi, \zeta_{\ell_{u}}\right) \\
u_{1} & =-\kappa \zeta_{\ell_{u}}+\Upsilon_{k}^{\prime}\left(\xi, \zeta_{\ell_{u}}\right) \\
\zeta_{\ell_{u}} & =\tilde{\xi}_{\ell_{u}}-\sum_{i=0}^{\ell_{u}-1} a_{i} g^{\ell_{u}-1} \tilde{\xi}_{i} .
\end{aligned}
$$

In order to obtain a feedback regulator only dependent on $\tilde{\xi}_{0}$ (namely of the form (33)), we follow [28] and design a "dirty derivatives" observer-based regulator

$$
\begin{aligned}
\dot{\tilde{\tilde{\xi}}}_{i} & =\hat{\tilde{\xi}}_{i+1}+L^{i+1} \lambda_{i}\left(\hat{\tilde{\xi}}_{0}-\tilde{\xi}_{0}\right) \quad i=0, \ldots \ell_{u}-1 \\
\dot{\tilde{\xi}}_{\ell_{u}} & =L^{\ell_{u}+1} \lambda_{r}\left(\hat{\tilde{\xi}}_{0}-\tilde{\xi}_{0}\right) \\
\dot{\xi} & =\Phi_{k}^{\prime}\left(\xi, \hat{\zeta}_{\text {sat }}\right) \\
u_{1} & =-\kappa \hat{\zeta}_{\text {sat }}+\Upsilon_{k}^{\prime}\left(\xi, \hat{\zeta}_{\text {sat }}\right)
\end{aligned}
$$

where $L$ is a positive design parameters, the $\lambda_{i}$ 's are such that the polynomial $s^{\ell_{u}}+\lambda_{\ell_{u}} s^{\ell_{u}-1}+\ldots+\lambda_{2} s+\lambda_{1}$ is Hurwitz and where

$$
\hat{\zeta}_{\text {sat }}=\operatorname{sat}_{\ell}\left(\hat{\tilde{\xi}}_{\ell_{u}}-g^{\ell_{u}-1} a_{0} \hat{\tilde{\xi}}_{1}-\ldots-g a_{\ell_{u}-1} \hat{\tilde{\xi}}_{\ell_{u}-1}\right)
$$

in which $\operatorname{sat}_{\ell}(s)$ is the saturation function such that $\operatorname{sat}_{\ell}(s)=$ $s$ if $|s| \leq \ell$ and $\operatorname{sat}_{\ell}(s)=\ell \operatorname{sgn}(s)$ otherwise. Letting $\tilde{\xi}=$ $\operatorname{col}\left(\tilde{\xi}_{1}, \ldots, \tilde{\xi}_{\ell_{u}}\right), \hat{\tilde{\xi}}=\operatorname{col}\left(\hat{\tilde{\xi}}_{1}, \ldots, \hat{\tilde{\xi}}_{\ell_{u}}\right)$, and defining the change of variables

$$
\hat{\tilde{\xi}} \mapsto e:=D_{L}(\tilde{\xi}-\hat{\tilde{\xi}})
$$

in which $D_{L}=\operatorname{diag}\left(L^{\ell_{u}-1}, \ldots, L, 1\right)$, it turns out that the overall closed-loop system (35), (41) reads as

$$
\begin{aligned}
\dot{x} & =\varphi(x)+\Delta_{1}(x, e) \\
\dot{e} & =L H e+\Delta_{2}(x, e)
\end{aligned}
$$

where $x:=\operatorname{col}\left(z, \zeta, \zeta_{\ell_{u}}, \xi\right), \dot{x}=\varphi(x)$ is a compact representation of (35), (40), $H$ is a Hurwitz matrix and $\Delta_{1}(\cdot)$ and $\Delta_{2}(\cdot)$ are defined as

$$
\Delta_{1}(\cdot)=\left(\begin{array}{c}
0 \\
0 \\
\kappa\left(\zeta_{\ell_{u}}-\hat{\zeta}_{\mathrm{sat}}\right)+\Upsilon_{k}^{\prime}\left(\xi, \hat{\zeta}_{\mathrm{sat}}\right)-\Upsilon_{k}^{\prime}\left(\xi, \zeta_{\ell_{u}}\right) \\
\Phi_{k}^{\prime}\left(\xi, \hat{\zeta}_{\mathrm{sat}}\right)-\Phi_{k}^{\prime}\left(\xi, \zeta_{\ell_{u}}\right)
\end{array}\right)
$$

and

$$
\Delta_{2}(\cdot)=\left(\begin{array}{c}
0 \\
\vdots \\
0 \\
\kappa\left(\zeta_{\ell_{u}}-\hat{\zeta}_{\mathrm{sat}}\right)+\Upsilon_{k}^{\prime}\left(\xi, \hat{\zeta}_{\mathrm{sat}}\right)-\Upsilon_{k}^{\prime}\left(\xi, \zeta_{\ell_{u}}\right)
\end{array}\right) .
$$

By construction the set graph $\tau^{\prime} \times\{0\}$ is $\operatorname{LES}(\mathcal{X})$ for the system $\dot{x}=\varphi(x)$ with $\mathcal{X}:=(\mathcal{Z} \times \mathcal{M}) \times \mathcal{M}_{\ell_{u}} \times \mathcal{N}^{\prime}$ and, by construction, it turns out that for any $\ell>0, \Delta_{1}(x, 0) \equiv 0$ and 
$\Delta_{2}(x, 0) \equiv 0$ for all $x \in$ graph $\tau^{\prime} \times\{0\}$. Furthermore, for any compact $\mathcal{M} \in \mathbb{R}^{\ell_{u}-1}, \mathcal{M}_{\ell_{u}} \in \mathbb{R}$ and $\hat{\mathcal{M}} \in \mathbb{R}^{\ell_{u}}$, there exists a compact set $\mathcal{E} \subset \mathbb{R}^{\ell_{u}}$ (dependent on $L$ ) such that if $\zeta(0) \in \mathcal{M}$, $\zeta_{\ell_{u}} \in \mathcal{M}_{\ell_{u}}$ and $\hat{\tilde{\xi}}(0) \in \hat{\mathcal{M}}$ then $e(0) \in \mathcal{E}$. Furthermore, by definition of saturation function, it turns out that for all $\bar{x}>0$ there exist $\delta_{1}>0$ and $\delta_{2}>0$ such that $\left|\Delta_{1}(x, e)\right| \leq \delta_{1}$ and $\Delta_{2}(x, e) \leq \delta_{2}$ for all $x,|x| \leq \bar{x}, e \in \mathbb{R}$ and $L>0$.

From these facts and by the result in [28], it follows that there exists a $L^{\star}>0$ such that for all $L \geq L^{\star}$ the set

$$
\begin{aligned}
& \operatorname{graph} \tau^{\prime} \times\{0\} \times\{0\}=\left\{\left((z, \zeta), \zeta_{\ell_{u}}, \xi, e\right) \in(\mathcal{R} \times\{0\})\right. \\
&\left.\times \mathbb{R} \times \mathbb{R}^{p} \times \mathbb{R}^{\ell_{u}}: \zeta_{\ell_{u}}=0, \xi=\tau^{\prime}(z, \zeta), e=0\right\}
\end{aligned}
$$

is $\operatorname{LES}\left(\mathcal{Z} \times \mathcal{M} \times \mathcal{M}_{\ell_{u}} \times \mathcal{N}^{\prime} \times \mathcal{E}\right)$.

From the previous results, the fact that $\mathcal{A}$ is LAS for the system $\dot{z}=A(z, \bar{u}(z))$, and the fact that on graph $\tau^{\prime} \times\{0\} \times\{0\}$ the closed-loop dynamics is described by $\dot{z}=A(z, \bar{u}(z)))$, the desired result follows by properly adapting the omega-limit set arguments used at the end of the proof of Theorem 2.

\section{Extended Dirty Derivatives Observer}

In this part we present a result which allows one to obtain a pure output feedback stabilizer once a partial state-feedback stabilizer (namely a stabilizer processing the output and a certain number of its time derivative) is known. Along the lines pioneered in [8] and [28], the idea is to substitute the knowledge of the time derivatives of the output with appropriate estimates provided by a "dirty derivative observer" (by using the terminology of [28]). In our context, though, we propose an "extended" dirty derivative observer, where the adjective "extended" is to emphasize the presence of a dynamic extension of the classical observer structure motivated by the need of handling the presence of possible not exponentially stable attractors in the partial-state feedback loop and the fact that, on this attractor, the measured output is not necessarily vanishing.

More specifically we assume, for the system (26), the existence of a dynamic stabilizer of the form

$$
\begin{aligned}
\dot{\varsigma} & =\bar{\varphi}\left(\varsigma, y, y^{(1)}, \ldots, y^{\left(n_{y}\right)}\right) \quad \varsigma \in \mathbb{R}^{d} \\
u & =\bar{\rho}\left(\varsigma, y, y^{(1)}, \ldots, y^{\left(n_{y}\right)}\right)
\end{aligned}
$$

such that the following property hold for the closed-loop system:

a) there exists a compact set $\mathcal{R} \supset \mathcal{A}$ and a continuous function $\tau: \mathcal{R} \rightarrow \mathbb{R}^{d}$ such that the sets $\operatorname{graph} \tau$ and graph $\left.\tau\right|_{\mathcal{A}}$ are respectively $\operatorname{LES}(\mathcal{Z} \times \mathcal{H})$ and $\operatorname{LAS}(\mathcal{Z} \times \mathcal{H})$ for the closed-loop system (26), (43) for some compact set $\mathcal{H} \subset \mathbb{R}^{d}$

b) there exist smooth functions $C_{i}, i=0, \ldots, n_{y}+1$, such that the output derivatives $y^{(i)}$ of the closed-loop system (26), (43) can be expressed as $y^{(i)}=C_{i}(z, \varsigma), i=$ $0, \ldots, n_{y}+1$ and the following holds:

$$
\left.\bar{\rho}\left(\varsigma, y, y^{(1)}, \ldots, y^{\left(n_{y}\right)}\right)\right|_{\text {graph } \tau}=\bar{u}(z) .
$$

Remark 6: Note that the previous conditions are automatically satisfied under the assumptions of Section III-A and by virtue of the results presented in the previous section. As a matter of fact, by bearing in mind (29) and Proposition 1 (and specifically (33)), the main outcome of the previous Section has been to design a dynamic controller of the form

$$
\begin{aligned}
\dot{\xi}_{0}= & \xi_{1} \\
& \vdots \\
\dot{\xi}_{\ell_{u}}= & \rho(\eta, \xi, \bar{u}(z)) \\
\dot{\eta}= & \varphi(\eta, \xi, \bar{u}(z)) \\
u & =\xi_{0}
\end{aligned}
$$

in which, according to (28) and to the definition of $\ell_{u}$

$$
\bar{u}(z)=\Psi\left(y, y^{(1)}, \ldots, y^{\left(n_{y}\right)}, \xi_{0}, \ldots, \xi_{n_{y}}\right) .
$$

System (44), (45) is clearly in the form (43) and, according to the result of Theorem 1, the previous conditions (a)-(b) are satisfied. $\triangleleft$

Within the previous framework we are able to prove the following proposition which, along with Proposition 1 and the previous remark, immediately yields Theorem 5 .

Proposition 2: Consider system (26) and assume the existence of a dynamic regulator of the form (43) such that the previous properties (a)-(b) are satisfied. Assume, in addition, that the triplet (31) is ISS-LER. Then there exist a positive $o$, a compact set $\mathcal{B} \subset \mathbb{R}^{o}$ and, for any compact set $\mathcal{N} \subset \mathbb{R}^{o}$, an output feedback controller of the form (32) such that the set $\mathcal{A} \times \mathcal{B}$ is $\operatorname{LAS}(\mathcal{Z} \times \mathcal{N})$ for the closed-loop system (26), (32).

Proof: As candidate controller, we consider a system of the form

$$
\begin{aligned}
\dot{\varsigma} & =\bar{\varphi}_{\ell}\left(\varsigma, y, \hat{y}_{1}, \ldots, \hat{y}_{n_{y}}\right) \\
\dot{\hat{y}}_{i} & =\hat{y}_{i+1}+L^{i+1} \lambda_{i}\left(\hat{y}_{0}-y\right) \quad i=0, \ldots, n_{y}-1 \\
\dot{\hat{y}}_{n_{y}} & =L^{n_{y}+1} \lambda_{n_{y}}\left(\hat{y}_{0}-y\right)+v \\
u & =\bar{\rho}_{\ell}\left(\varsigma, y, \hat{y}_{1}, \ldots, \hat{y}_{n_{y}}\right)
\end{aligned}
$$

in which $v$ is a control input to be designed, $L$ is a positive design parameters, the $\lambda_{i}$ 's are the coefficients of an Hurwitz polynomial and $\bar{\varphi}_{\ell}(\cdot)$ and $\bar{\rho}_{\ell}$ are appropriate saturated versions of the functions $\bar{\varphi}(\cdot)$ and $\bar{\rho}(\cdot)$ of (43) satisfying $\bar{\varphi}_{\ell}(s)=\bar{\varphi}(s)$ if $|\bar{\varphi}(s)| \leq \ell,\left|\bar{\varphi}_{\ell}(s)\right| \leq \ell$ for all $s$, and $\bar{\rho}_{\ell}(s)=\bar{\rho}(s)$ if $|\bar{\rho}(s)| \leq \ell$, $\left|\bar{\rho}_{\ell}(s)\right| \leq \ell$ for all $s$, with $\ell$ a design parameter.

Let now $y_{d}=\operatorname{col}\left(y, y^{(1)}, \ldots, y^{\left(n_{y}\right)}\right), \hat{y}=$ $\operatorname{col}\left(\hat{y}_{0}, \hat{y}_{1}, \ldots, \hat{y}_{n_{y}}\right)$ and consider the change of variables $\hat{y} \mapsto e=D_{L}\left(y_{d}-\hat{y}\right)$ where $D_{L}=\operatorname{diag}\left(L^{n_{y}}, L^{n_{y}-1}, \ldots, 1\right)$. In this coordinate setting, by denoting $x=\operatorname{col}(z \varsigma)$, the overall closed-loop system reads as

$$
\begin{aligned}
& \dot{x}=f(x)+\Delta(x, e) \\
& \dot{e}=L H e+B(q(x)+v)
\end{aligned}
$$

in which $H$ is a Hurwitz matrix in observability canonical form, $B=(0 \ldots 01)^{\mathrm{T}} \dot{x}=f(x)$ is a compact representation of the system (26), (43), $q(x)=C_{n_{y}+1}(z, \varsigma)$ and

$\Delta(x, e)=$

$\left(\begin{array}{c}0 \\ A\left(z, \bar{\rho}_{\ell}\left(\varsigma, y, \hat{y}_{1}, \ldots, \hat{y}_{n_{y}}\right)\right)-A\left(z, \bar{\rho}\left(\varsigma, y, y^{(1)}, \ldots, y^{\left(n_{y}\right)}\right)\right) \\ \left.\left.\bar{\varphi}_{\ell}\left(\varsigma, y, \hat{y}_{1}, \ldots, \hat{y}_{n_{y}}\right)\right)-\bar{\varphi}\left(\varsigma, y, y^{(1)}, \ldots, y^{\left(n_{y}\right)}\right)\right)\end{array}\right)$

in which, in the latter, we have left the "original" coordinates $\hat{y}_{i}$ for notational convenience. 
By definition of $\Delta$, of $\bar{\rho}_{\ell}$ and of $\bar{\varphi}_{\ell}$, it turns out that for any bounded set $\mathcal{Z}_{M}^{\prime}$ and $\mathcal{H} \subset \mathbb{R}^{p+\nu}$, there exists an $\ell^{\star}$ such that for any $\ell \geq \ell^{\star}$

$$
\Delta(x, 0)=0 \quad \forall x \in \mathcal{Z}_{M}^{\prime} \times \mathcal{H} .
$$

Furthermore, by the fact that the triplet (31) is ISS-LER it follows that there exists a compact set $\mathcal{R}^{\prime} \supset \mathcal{A}$ which is LES for $\dot{z}=A(z, \bar{u}(z))$ with $\mathcal{D}\left(\mathcal{R}^{\prime}\right) \supseteq \mathcal{D}(\mathcal{A})$. Let $\mathcal{R}^{\prime \prime}:=\mathcal{R} \cap \mathcal{R}^{\prime}$. By item (a) above and by going throughout the proof of Proposition 1 , it turns out that graph $\left.\tau\right|_{\mathcal{R}^{\prime \prime}}$ is LES for $\dot{x}=f(x)$. Moreover, the set $\mathcal{R}^{\prime \prime}$ is LES for $\dot{z}=A(z, \bar{u}(z))$ with $\mathcal{D}\left(\mathcal{R}^{\prime \prime}\right) \supseteq \mathcal{D}(\mathcal{A})$ and, by definition of ISS-LER, it is possible to claim that the triplet $\left(A(z, \bar{u}(z)), L_{A(z, \bar{u}(z))}^{\left(n_{y}+1\right)} C(z), \mathcal{R}^{\prime \prime}\right)$ is ISS-LER. From the previous facts, from the item (b) above, which implies that

$$
\left.q(x)\right|_{\text {graph }\left.\tau\right|_{\mathcal{R}^{\prime \prime}}}=C_{n_{y}+1}(z, \tau(z))=L_{A(z, \bar{u}(z))}^{\left(n_{y}+1\right)} C(z)
$$

and by Proposition 5 in Appendix A, it follows that the triplet

$$
\left(f(x), q(x),\left.\operatorname{graph} \tau\right|_{\mathcal{R}^{\prime \prime}}\right)
$$

is LER. Thus system (46) fits into the framework of Theorem 2 (with graph $\left.\tau\right|_{\mathcal{R}^{\prime \prime}}$ playing the role of the set $\mathcal{A}$ ) by which the result follows (by using the fact that graph $\left.\tau\right|_{\mathcal{A}}$ is LAS for $\dot{x}=f(x)$ and by adapting the omega-limit set arguments at the end of the proof of Theorem 2).

\section{SUFFICIENT CONDITIONS FOR EXPONENTIAL REPRODUCIBILITY}

Having established with Theorems 2 and 5 the interest of local exponential reproducibility for solving the problem of (robust) output feedback stabilization via a locally Lipschitz regulator, in this section we present a few results which are useful to test when a triplet $(F, Q, \mathcal{A})$ is ISS-LER (and thus LER) and, eventually, to design the functions $(\varphi, \psi, \gamma)$.

As also commented in Section II-C, the first requirement behind the definition is the existence of a compact set $\mathcal{R} \supseteq \mathcal{A}$ which is LES for $\dot{z}=F(z)$. In this respect we present a result which claims that the existence of a set $\mathcal{R}$ which is LES for $\dot{z}=F(z)$ is automatically guaranteed if the set $\mathcal{A}$ is LAS for $\dot{z}=F(z)$. Thus, put in the context of Theorem 2 , the first requirement of the definition is not restrictive at all. Details of this fact are reported in the following proposition whose proof can be found in [23].

Lemma 1: Consider system

$$
\dot{z}=F(z) \quad z \in \mathbb{R}^{m}
$$

evolving on an invariant closed set $\mathcal{C}_{m} \subset \mathbb{R}^{m}$. Let $\mathcal{A} \subset \mathcal{C}_{m}$ be a compact set which is LAS with domain of attraction $\mathcal{D}(\mathcal{A}) \subseteq$ $\mathcal{C}_{m}$. For any compact set $\mathcal{S} \subset \mathcal{C}_{m}$ such that $\mathcal{A} \subset$ int $\mathcal{S}$, there exists a compact set $\mathcal{R}$ satisfying $\mathcal{A} \subseteq \mathcal{R} \subset \mathcal{S}$ which is LES for (47) with domain of attraction $\mathcal{D}(\mathcal{R}) \equiv \mathcal{D}(\mathcal{A})$.

Remark 7: By going throughout the proof of the Lemma in [23], it is easily realized that the set $\mathcal{R}$ is nothing else but any sub-level set of the Lyapunov function associated to the locally asymptotically stable set $\mathcal{A}$ of (47) contained in $\mathcal{S} . \triangleleft$

We pass now to analyze the second crucial requirement behind the definition of ISS-LER, namely the existence of locally
Lipschitz functions $(\varphi, \psi, \gamma)$ and $T$ such that conditions (8) and (12) are satisfied for system (11). Since the property in question is related to the ability of reproducing any signal $Q(z(t))$ generated by the system $\dot{z}(t)=F(z(t))$ by taking its initial conditions in the set $\mathcal{R}$, it is not surprising that the theory of nonlinear output regulation, and specifically the design techniques proposed in the related literature to construct internal models, can be successfully used to this purpose (see [21]). In particular, in the following, we present a technique which is directly taken, with minor adaptations, from the literature of output regulation. In general it is worth noting how any method to design locally Lipschitz internal models can be effectively used for this scope (see, besides others, [13], [14]).

By following [6] we present a method which draws its inspiration from high-gain design techniques of nonlinear observers. Specifically, it is possible to state the following proposition which comes from Lemma 1 and from minor adaptations ${ }^{3}$ of the main result of [6] (see the quoted work for the proof).

Proposition 3: Let $F: \mathbb{R}^{m} \rightarrow \mathbb{R}^{m}$ and $Q: \mathbb{R}^{m} \rightarrow \mathbb{R}$ be given smooth functions and $\mathcal{A} \subset \mathbb{R}^{m}$ be a given compact set which is LAS for $\dot{z}=F(z)$. Assume, in addition, that there exist a $\tilde{m}>0$, a compact set $\mathcal{S}$ such that $\mathcal{A} \subset$ int $\mathcal{S}$ and a locally Lipschitz function $f: \mathbb{R}^{\tilde{m}} \rightarrow \mathbb{R}$ such that the following holds:

$L_{F(z)}^{\tilde{m}} Q(z)=f\left(Q(z), L_{F(z)} Q(z), \ldots, L_{F(z)}^{\tilde{m}-1} Q(z)\right) \forall z \in \mathcal{S}$.

Then the triplet $(F, Q, \mathcal{A})$ is ISS-LER. In particular $(\varphi, \psi, \gamma)$ can be taken as the functions $\varphi: \mathbb{R}^{\tilde{m}} \rightarrow \mathbb{R}^{\tilde{m}}, \psi: \mathbb{R}^{\tilde{m}} \rightarrow \mathbb{R}^{\tilde{m}}$, $\gamma: \mathbb{R}^{\tilde{m}} \rightarrow \mathbb{R}$ defined as

$$
\begin{aligned}
& \varphi(\xi)=\left(\begin{array}{c}
\xi_{2}+\lambda_{0} L \xi_{1} \\
\xi_{3}+\lambda_{1} L^{2} \xi_{1} \\
\vdots \\
\xi_{\tilde{m}}+\lambda_{\tilde{m}-2} L^{\tilde{m}-1} \xi_{1} \\
f_{c}\left(\xi_{1}, \xi_{2}, \ldots, \xi_{\tilde{m}}\right)+\lambda_{m-1} L^{\tilde{m}} \xi_{1}
\end{array}\right), \\
& \psi(\xi)=-\left(\begin{array}{lllll}
\lambda_{0} L & \lambda_{1} L^{2} & \cdots & \lambda_{\tilde{m}-2} L^{\tilde{m}-1} & \lambda_{\tilde{m}-1} L^{\tilde{m}}
\end{array}\right)^{\mathrm{T}}
\end{aligned}
$$

and $\gamma(\xi)=-\xi_{1}$, where $L$ is a positive design parameter to be taken sufficiently large, $\lambda_{i}, i=0, \ldots, \tilde{m}-1$, are such that the polynomial $s^{\tilde{m}}+\lambda_{\tilde{m}-1} s^{\tilde{m}-1}+\ldots+\lambda_{1} s+\lambda_{0}$ is Hurwitz, and $f_{c}(\cdot)$ is any bounded function such that $f_{c} \circ \tau(z)=f(z)$ for all $z \in \mathcal{S}$ where $\tau: \mathbb{R}^{m} \rightarrow \mathbb{R}^{\tilde{m}}$ is defined as

$$
\tau(z)=\left(\begin{array}{lll}
Q(z) & L_{F(z)} Q(z) & \cdots L_{F(z)}^{\tilde{m}-1} Q(z)
\end{array}\right)^{\mathrm{T}} .
$$

Example: Consider the case in which $z:=\operatorname{col}(w, x)$, $F(z):=\operatorname{col}\left(0,-x^{3}+w\right), Q(z):=x, W:=[\underline{w}, \bar{w}]$ and $\mathcal{A}$ is the set

$$
\mathcal{A}:=\left\{(w, x) \in \mathcal{C}_{1}: x=\sqrt[3]{w}\right\}
$$

with $\mathcal{C}_{1}:=W \times \mathbb{R}$, which is LAS for $\dot{z}=F(z)$. By Lemma 1 , and Proposition 3 it is possible to prove that triplet $(F(z), Q(z), \mathcal{A})$ is ISS-LER. As a matter of fact, let $\mathcal{S}$ be a compact set of the form

$$
\mathcal{S}=\left\{(w, x) \in \mathcal{C}_{1}: \sqrt[3]{\underline{w}}-\epsilon \leq x \leq \sqrt[3]{\bar{w}}+\epsilon\right\}
$$

${ }^{3}$ The adaptation consists only in proving the ISS property which is behind the definition of ISS-LER. 
with $\epsilon>0$, so that $\mathcal{A} \subset \operatorname{int} \mathcal{S}$, and note that, by Lemma 1, there exists a set $\mathcal{R}, \mathcal{A} \subseteq \mathcal{R} \subset \operatorname{int} \mathcal{S}$, which is LES with $\mathcal{D}(\mathcal{R})=$ $\mathcal{D}(\mathcal{A})$. Furthermore it is easy to check that

$$
L_{F(z)}^{2} Q(z)=-3 Q^{2}(z) L_{F(z)} Q(z) \quad \forall z \in \mathcal{C}_{1}
$$

namely condition (48) holds (with $\tilde{m}=2$ ), and thus the triplet $(F(z), Q(z), \mathcal{A})$ is ISS-LER. According to the proposition, the functions $(\varphi, \psi, \gamma)$ can be designed as

$$
\varphi(\xi)=\left(\begin{array}{c}
\xi_{2}+\lambda_{0} L \xi_{1} \\
f_{c}\left(\xi_{1}, \xi_{2}\right)+\lambda_{1} L^{2} \xi_{1}
\end{array}\right) \quad \psi(\xi)=\left(\begin{array}{c}
-\lambda_{0} L \\
-\lambda_{1} L^{2}
\end{array}\right)
$$

and $\gamma(\xi)=-\xi_{1}$, where $\lambda_{0}$ and $\lambda_{1}$ are such that $s^{2}+\lambda_{1} s+$ $\lambda_{0}$ is an Hurwitz polynomial, $L$ is a sufficiently large design parameter and $f_{c}(\cdot)$ is any smooth bounded function such that

$$
f_{c}\left(x,-x^{3}+w\right)=-3 x^{2}\left(-x^{3}+w\right) \quad \forall(x, w) \in \mathcal{S} .
$$

We observe that in the particular case $\underline{w} \equiv \bar{w}=0$, namely $w \equiv$ 0 , property (48) holds with $\tilde{m}=1$ as $L_{F(z)} Q(z)=-Q^{3}(z)$. As the consequence, the functions $(\varphi, \psi, \gamma)$ can be simply taken equal to $\varphi(\xi)=-\operatorname{sat}\left(\xi^{3}\right), \psi(\xi)=-L$ and $\gamma(\xi)=-\xi, \xi \in \mathbb{R}$, where $\operatorname{sat}(\cdot)$ is a saturation function whose saturation level can be taken arbitrary (by consequently selecting the parameter $\epsilon$ in the previous analysis). This and Theorem 2 yield the controller (2) in the example of Section II-A. $\triangleleft$

Remark 8: It is well-known (see, for instance, [11]) that a sufficient condition for a pair $(F, Q)$ to satisfy property (48) locally with respect to a point $z_{0}$ is that its observability distribution at $z_{0}$ (see [12])

$$
\Omega_{m}(z)=\sum_{k=0}^{m-1} \operatorname{span} \frac{\partial}{\partial z} L_{F(z)}^{k} Q(z)
$$

has dimension $m$ at $z=z_{0}$, namely if the system $\dot{z}=F(z)$ with output $y_{z}=Q(z)$ satisfies the observability rank condition (by using the terminology of [12]) at $z_{0}$. Such a condition represents an observability condition for the system $\dot{z}=F(z)$ with output $y_{z}=Q(z)$ which, however, is far to be necessary to fulfill the property of ISS-LER. In this respect it must be stressed again that the property of local exponential reproducibility does not involve any state observability property of system $\dot{z}=F(z)$ with output $y_{z}=Q(z)$ but rather a property of output reproducibility. $\triangleleft$

\section{EXAMPLES}

\section{A. Example 1}

We consider the system presented in [4] given by

$$
\begin{aligned}
\dot{x}_{1} & =x_{2} \\
\dot{x}_{2} & =-x_{1} e^{x_{1} x_{2}}+y \\
\dot{y} & =x_{2}+u
\end{aligned}
$$

with input $u \in \mathbb{R}$ and measurable output $y \in \mathbb{R}$, and the problem of stabilizing the origin by means of a locally Lipschitz output feedback. The zero dynamics of the system, described by

$$
\dot{x}_{1}=x_{2} \quad \dot{x}_{2}=-x_{1} e^{x_{1} x_{2}}
$$

have the origin which is globally asymptotically stable but not locally exponentially (the linear approximation at the origin has a pair of purely imaginary eigenvalues). As shown in [4], the static output feedback $u=-k y$, for any positive $k$ fails to stabilize the origin of the closed-loop system, because the resulting linear approximation at the origin is unstable. In particular, for any $r>0$, there is $k^{*}>0$ such that, if $k>k^{*}$, the closed-loop system obtained with $u=-k y$ has an unstable equilibrium at the origin and a stable limit cycle entirely contained in the sphere of radius $r$ centered at the origin.

Nevertheless, we show how the theory proposed in the paper (and specifically Theorem 2) can be used to design a dynamic output feedback regulator stabilizing the origin. In particular, by letting $u=-\kappa y+v$, it turns out that system (51) can be written in the form (7) with $x=\left(x_{1}, x_{2}\right)^{\mathrm{T}}, B=-A=1$ $f(x, y)=\operatorname{col}\left(x_{2},-x_{1} e^{x_{1} x_{2}}+y\right), q(x, y)=x_{2}$. In particular the minimum-phase assumption in Section II-B is fulfilled with $\mathcal{A}=0$. In order to apply Theorem 2 , the local exponential reproducibility of the triplet $(f(x, 0), q(x, 0), \mathcal{A})$ must be checked. To this purpose we show that Proposition 3 applies. For, note that $L_{f(x, 0)} q(x, 0)=-x_{1} e^{x_{1} x_{2}}$ and that the transformation

$$
\left(x_{1}, x_{2}\right) \rightarrow\left(x_{2},-x_{1} e^{x_{1} x_{2}}\right)=\left(q(x, 0), L_{f(x, 0)} q(x, 0)\right)
$$

is a local diffeomorphism at the origin, namely system (52) with output $x_{2}$ is locally observable at the origin. From the previous fact it turns out that there exists a smooth function $\Upsilon: \mathbb{R}^{2} \rightarrow \mathbb{R}$ and a compact set $\mathcal{S}$, including $\mathcal{A}$ in its interior, such that

$$
L_{f(x, 0)}^{2} q(x, 0)=\Upsilon\left(q(x, 0), L_{f(x, 0)} q(x, 0)\right) \quad \forall x \in \mathcal{S} .
$$

Hence, by Proposition 3, the triplet $(f(x, 0), q(x, 0), \mathcal{A})$ is ISS-LER (and thus LER) and Theorem 2 guarantees the existence of the locally Lipschitz output feedback dynamic regulator. In particular the regulator has the form

$$
\begin{aligned}
\dot{\eta}_{1} & =\eta_{2}+c_{0} L \kappa y \\
\dot{\eta}_{2} & =\Upsilon_{c}\left(\eta_{1}, \eta_{2}\right)+c_{1} L^{2} \kappa y \\
v & =-\eta_{1}
\end{aligned}
$$

where $c_{0}, c_{1}$ are arbitrary coefficients such that $s^{2}+c_{1} s+c_{0}$ is Hurwitz, $\Upsilon_{c}$ is an arbitrary bounded function such that $\Upsilon_{c} 0$ $\tau(z)=\Upsilon(z)$ for all $z \in \mathcal{S}$ with $\tau(z)=\left(x_{2},-x_{1} e^{x_{1} x_{2}}\right)^{\mathrm{T}}$, and $L$ and $\kappa(L)$ are positive numbers to be taken sufficiently large according to the desired domain of attraction of the closed-loop system.

\section{B. Example 2}

We consider the set-point control problem in which the output $\zeta_{1}$ of the system in $\mathbb{R}^{3}$

$$
\begin{aligned}
\dot{z} & =-z|z|+\zeta_{1} \\
\dot{\zeta}_{1} & =\zeta_{2} \\
\dot{\zeta}_{2} & =z+u
\end{aligned}
$$

with control input $u \in \mathbb{R}$, is required to track a constant set point $w$ by only processing the regulation error $\zeta_{1}-w$. The problem can be cast as the problem formulated in Section II-B. 
In particular, let $e=\zeta_{1}-w$ and $y=e+\dot{e}=\zeta_{1}+\zeta_{2}-y$ so that system (53) reads as

$$
\begin{aligned}
& \dot{z}=-z|z|+w+e \\
& \dot{e}=-e+y \\
& \dot{y}=y-e_{1}+z+u
\end{aligned}
$$

with the trivial dynamics of the set-point $w$ governed by the exosystem $\dot{w}=0$. By letting $u=-\kappa y+v$ the previous system is in the form (7) with $x=\operatorname{col}(w, z, e), f(x, y)=\operatorname{col}(0,-z|z|+$ $w+e,-e+y), q(x, y)=y-e_{1}+z$ and $A=-B=-1$. Furthermore, elementary arguments can be used to prove that the minimum-phase assumption of Section II-B is satisfied. More specifically, denoting by $W=[\underline{w}, \bar{w}]$ the closed interval containing the value of $w$, it turns out that the set $\mathcal{A}=\mathcal{A}^{\prime} \times\{0\}$ with

$$
\mathcal{A}^{\prime}=\{(w, z) \in W \times \mathbb{R}: z|z|=w\}
$$

is asymptotically stable for $\dot{x}=f(x, 0)$ with domain of attraction $\mathcal{D}(\mathcal{A})=W \times \mathbb{R} \times \mathbb{R}$, but not locally exponentially stable. The minimum-phase assumption of Section II-B is thus satisfied and, by Theorem 2, a feedback regulator of the form (6) with $y_{m}=y$ exists if the triplet $(f(x, 0), q(x, 0), \mathcal{A})$ is LER. To check if this is the case, let $f^{\prime}(w, z)=\operatorname{col}(0,-z|z|+$ $w), q^{\prime}(w, z)=z$ and note that, by employing the triangular structure of $f(x, 0)$ and by Proposition 5 in the Appendix, it turns out that the triplet $(f(x, 0), q(x, 0), \mathcal{A})$ is LER if the triplet $\left(f^{\prime}(w, z), q^{\prime}(w, z), \mathcal{A}^{\prime}\right)$ is ISS-LER. Since

$$
L_{f^{\prime}(w, z)}^{2} q^{\prime}(w, z)=-\left|q^{\prime}(w, z)\right| L_{f^{\prime}(w, z)} q^{\prime}(w, z)
$$

$\forall(w, z) \in \mathbb{R} \times \mathbb{R}$, by Proposition 3 , the triplet $\left(f^{\prime}(w, z), q^{\prime}(w, z), \mathcal{A}^{\prime}\right)$ is ISS-LER and thus the problem at hand has a solution. In particular, by going throughout the proof of Proposition 5 and by bearing in mind the specific expression of the triplet $(\varphi, \psi, \gamma)$ in Proposition 3, it turns out that the regulator has the form

$$
\begin{aligned}
\dot{\xi}_{1} & =\xi_{2}+c_{0} L \kappa y \\
\dot{\xi}_{2} & =\Upsilon_{c}\left(\xi_{1}, \xi_{2}\right)+c_{1} L^{2} \kappa y \\
u & =-\kappa y-\xi_{1}
\end{aligned}
$$

in which $\Upsilon_{c}$ is any smooth bounded function such that

$$
\Upsilon_{c}\left(\xi_{1}, \xi_{2}\right)=-\left|\xi_{1}\right| \xi_{2}
$$

for all $\left(\xi_{1}, \xi_{2}\right)$ such that $\xi_{1} \in\left[\min _{(w, z) \in \mathcal{A}^{\prime}}\{z\}-\epsilon, \max _{(w, z) \in \mathcal{A}^{\prime}}\{z\}+\right.$ $\epsilon]$ and $\left|\xi_{2}\right| \leq \epsilon$, with $\epsilon$ a positive number, $c_{0}, c_{1}$ are arbitrary coefficients such that $s^{2}+c_{1} s+c_{0}$ is Hurwitz, $L$ is a design parameter. The controller thus obtained depends on $y=e+$ $\dot{e}$, namely only the error $e$ and its time derivative. In order to obtain a pure error feedback regulator, by following the proof of Proposition 1, it is possible to substitute the term $y$ in (54) with the (saturated) estimate

$$
\hat{y}=\operatorname{sat}_{\ell}\left(\hat{e}_{1}+\hat{e}_{2}\right)
$$

where $\operatorname{sat}_{\ell}$ is a saturation function and $\left(\hat{e}_{1}, \hat{e}_{2}\right)$ are the state variables of the "dirty derivatives observer"

$$
\begin{aligned}
& \dot{\hat{e}_{1}}=\hat{e}_{2}+K \lambda_{0}\left(\hat{e}_{1}-e\right) \\
& \dot{\hat{e}_{2}}=K^{2} \lambda_{1}\left(\hat{e}_{1}-e\right)
\end{aligned}
$$

with $c_{0}, c_{1}$ arbitrary coefficients such that $s^{2}+c_{1} s+c_{0}$ is Hurwitz and $K$ a positive design parameter. The high-gain design parameters $L, \kappa(L)$, and $K(\kappa)$ must be taken sufficiently large according to the desired domain of attraction for the closed-loop system.

\section{CONCLUSION}

The problem of smoothly stabilizing, by output feedback, nonlinear systems whose zero dynamics have an asymptotically but not exponentially stable attractor has been considered. The idea developed in the paper is to approach the problem as a set stabilization problem by properly using high-gain arguments and design tools commonly employed in the context of nonlinear output regulation. It has been shown how to design locally Lipschitz dynamic stabilizers embedding an "internal model" of the zero dynamics close to the asymptotic attractor in order to overtake the lack of exponential stability and enforcing the desired asymptotic properties. The proposed design procedure contained in Theorem 2 relies upon a property of Local Exponential Reproducibility which has been characterized in terms of sufficient conditions in Section IV. As possible applications of the proposed tool it has been shown how to overtake local exponential stability requirements in backstepping procedures and design of robust observers conventionally adopted in robust output feedback stabilization.

\section{APPENDIX}

\section{A. Auxiliary Results}

Proposition 4: Let

$$
\dot{z}=F(z)
$$

be a given locally Lipschitz system and let $S$ be a compact set which is forward invariant for (55) and which uniformly (in the initial condition) attracts the trajectories of (55) originating in a compact set $D \supset S$. Then $\omega(D)=\omega(S) \subseteq S$.

Proof: First of all note that $\omega(D)$ and $\omega(S)$ exist and that, by definition, $\omega(S) \subseteq \omega(D)$. Furthermore $\omega(S) \subseteq S$ as $S$ is forward invariant for (55). To prove that $\omega(D)=\omega(S)$ suppose that it is not, namely that there exist a $\bar{z} \in \omega(D)$ and an $\epsilon>0$ such that $|\bar{z}|_{S} \geq \epsilon$. As $S$ uniformly attracts the trajectories of (55) originating from $D$, there exists a $t_{\epsilon / 2}>0$ such that $\left|z\left(t, z_{0}\right)\right|_{S} \leq \epsilon / 2$ for all $z_{0} \in D$ and for all $t \geq t_{\epsilon / 2}$. Moreover, by definition of $\omega(D)$, there exist sequences $\left\{z_{n}\right\}_{0}^{\infty}$ and $\left\{t_{n}\right\}_{0}^{\infty}$, with $z_{n} \in D$ and $\lim _{n \rightarrow \infty} t_{n}=\infty$, and (without loss of generality) $t_{0} \geq t_{\epsilon / 2}$, such that $\lim _{n \rightarrow \infty} z\left(t_{n}, z_{n}\right)=\bar{z}$. This, in particular, implies that for any $\nu>0$ there exists a $n_{\nu}>0$ such that $\left|z\left(t_{n}, z_{n}\right)-\bar{z}\right| \leq \nu$ for all $n \geq n_{\nu}$. But, by taking $\nu=\epsilon / 2$, this contradicts that $S$ uniformly attracts the trajectories of the system originating from $D$. 
Proposition 5: Consider a system of the form

$$
\begin{array}{ll}
\dot{x}_{1}=f_{1}\left(x_{1}, x_{2}\right) & x_{1} \in \mathbb{R}^{n_{1}} \\
\dot{x}_{2}=f_{2}\left(x_{1}, x_{2}\right) & x_{2} \in \mathbb{R}^{n_{2}}
\end{array}
$$

and assume that there exist a compact set $\mathcal{A} \subset \mathbb{R}^{n_{1}}$ and a smooth function $\tau: \mathbb{R}^{n_{1}} \rightarrow \mathbb{R}^{n_{2}}$ such that the set

$$
\left.\operatorname{graph} \tau\right|_{\mathcal{A}}=\left\{\left(x_{1}, x_{2}\right) \in \mathcal{A} \times \mathbb{R}^{n_{2}} \quad: \quad x_{2}=\tau\left(x_{1}\right)\right\}
$$

is LES for (56) and the set $\mathcal{A}$ is LES for the system $\dot{x}_{1}=$ $f_{1}\left(x_{1}, \tau\left(x_{1}\right)\right)$. Let $q: \mathbb{R}^{n_{1}} \times \mathbb{R}^{n_{2}} \rightarrow \mathbb{R}$ be a smooth function. If the triplet $\left(f_{1}\left(x_{1}, \tau\left(x_{1}\right)\right), q\left(x_{1}, \tau\left(x_{1}\right)\right), \mathcal{A}\right)$ is ISS-LER then the triplet $\left(\operatorname{col}\left(f_{1}\left(x_{1}, x_{2}\right), f_{2}\left(x_{1}, x_{2}\right)\right), q\left(x_{1}, x_{2}\right)\right.$, graph $\left.\left.\tau\right|_{\mathcal{A}}\right)$ is LER.

Proof: Let $\bar{f}_{1}\left(x_{1}\right)=f_{1}\left(x_{1}, \tau\left(x_{1}\right)\right)$ and $\bar{q}\left(x_{1}\right)=$ $q\left(x_{1}, \tau\left(x_{1}\right)\right)$. Since $\mathcal{A}$ is LES for $\dot{x}_{1}=\bar{f}_{1}\left(x_{1}\right)$ and the triplet $\left(\bar{f}_{1}\left(x_{1}\right), \bar{q}\left(x_{1}\right), \mathcal{A}\right)$ is ISS-LER, for any compact set $\bar{X}_{1} \subset \mathcal{D}(\mathcal{A})$, there exist an integer $\bar{p}$ and locally Lipschitz functions $\bar{\varphi}: \mathbb{R}^{\bar{p}} \rightarrow \mathbb{R}^{\bar{p}}, \bar{\psi}: \mathbb{R}^{\bar{p}} \rightarrow \mathbb{R}, \bar{\gamma}: \mathbb{R}^{\bar{p}} \rightarrow \mathbb{R}$ and $\bar{T}: \mathbb{R}^{n_{1}} \rightarrow \mathbb{R}^{\bar{p}}$ such that, for all $\bar{x}_{10} \in \bar{X}_{1}$ and $\bar{\xi}_{0} \in \mathbb{R}^{\bar{p}}$ and for all locally essentially bounded $v(t)$, the solution $\left(\bar{x}_{1}(t), \bar{\xi}(t)\right)$ of the system

$$
\begin{array}{rlr}
\dot{\bar{x}}_{1} & =\bar{f}_{1}\left(\bar{x}_{1}\right) \quad \bar{x}_{1}(0)=\bar{x}_{10} \\
\dot{\bar{\xi}} & =\bar{\varphi}(\bar{\xi})+\bar{\psi}(\bar{\xi})\left[\bar{q}\left(\bar{x}_{1}\right)+v\right] \quad \bar{\xi}(0)=\bar{\xi}_{0}
\end{array}
$$

satisfies

$$
\begin{array}{r}
\left|\left(\bar{x}_{1}(t), \bar{\xi}(t)\right)\right|_{\left.\operatorname{graph} \bar{T}\right|_{\mathcal{A}}} \leq \beta_{1}\left(t,\left|\left(\bar{x}_{10}, \bar{\xi}_{0}\right)\right|_{\left.\operatorname{graph} \bar{T}\right|_{\mathcal{A}}}\right) \\
+\ell\left(\sup _{\tau \leq t}|v(\tau)|\right)
\end{array}
$$

where $\beta_{1}(\cdot, \cdot)$ and $\ell(\cdot)$ are respectively a locally exponential class- $\mathcal{K} \mathcal{L}$ and a class- $\mathcal{K}$ functions, and

$$
\bar{\gamma}\left(\bar{T}\left(\bar{x}_{1}\right)\right)+\bar{q}\left(\bar{x}_{1}\right)=0 \quad \forall \bar{x}_{1} \in \mathcal{A} .
$$

Furthermore, by the assumption that graph $\left.\tau\right|_{\mathcal{A}}$ is LES for (56), for any $\left(x_{10}, x_{20}\right) \in \mathcal{D}\left(\right.$ graph $\left.\left.\tau\right|_{\mathcal{A}}\right)$ the solution $\left(x_{1}(t), x_{2}(t)\right)$ of (56) with initial condition $\left(x_{1}(0), x_{2}(0)\right)=\left(x_{10}, x_{20}\right)$ satisfies

$$
\left|\left(x_{1}(t), x_{2}(t)\right)\right|_{\left.\operatorname{graph} \tau\right|_{\mathcal{A}}} \leq \beta_{2}\left(t,\left|\left(x_{10}, x_{20}\right)\right|_{\left.\operatorname{graph} \tau\right|_{\mathcal{A}}}\right)
$$

where $\beta_{2}(\cdot, \cdot)$ is a locally exponential class- $\mathcal{K} \mathcal{L}$ function.

Now pick a compact set $\bar{X}_{1} \subset \mathcal{D}(\mathcal{A})$ and the functions $(\bar{\varphi}(\cdot), \bar{\psi}(\cdot), \bar{\gamma}(\cdot))$ accordingly, and denote by $\left(x_{1}(t), x_{2}(t), \xi(t)\right)$ the solution of the system

$$
\begin{aligned}
\dot{x}_{1} & =f_{1}\left(x_{1}, x_{2}\right) \quad x_{1} \in \mathbb{R}^{n_{1}} \\
\dot{x}_{2} & =f_{2}\left(x_{1}, x_{2}\right) \quad x_{2} \in \mathbb{R}^{n_{2}} \\
\dot{\xi} & =\bar{\varphi}(\xi)+\bar{\psi}(\xi) q\left(x_{1}, x_{2}\right) \quad \xi \in \mathbb{R}^{\bar{p}}
\end{aligned}
$$

with initial conditions $\left(x_{10}, x_{20}, \xi_{0}\right) \in \mathbb{R}^{n_{1}} \times \mathbb{R}^{n_{2}} \times \mathbb{R}^{\bar{p}}$ at time $t=0$. Let $X \subset \mathbb{R}^{n_{1}} \times \mathbb{R}^{n_{2}}$ be an arbitrary compact set such that $X \subset \mathcal{D}\left(\right.$ graph $\left.\left.\tau\right|_{\mathcal{A}}\right)$, let $\mathcal{R}:=$ graph $\left.\tau\right|_{\mathcal{A}}$ and let $T: \mathbb{R}^{n_{1}} \times \mathbb{R}^{n_{2}} \rightarrow \mathbb{R}^{\bar{p}}$ be the locally Lipschitz function defined as $T\left(x_{1}, x_{2}\right)=\bar{T}\left(x_{1}\right)$. We shall prove in the following that for any initial condition $\left(x_{10}, x_{20}, \xi_{0}\right) \in X \times \mathbb{R}^{\bar{p}}$ the trajectory $\left(x_{1}(t), x_{2}(t), \xi(t)\right)$ of (59) satisfies

$\left|\left(x_{1}(t), x_{2}(t), \xi(t)\right)\right|_{\left.\operatorname{graph} T\right|_{\mathcal{R}}} \leq \beta_{3}\left(t,\left|\left(x_{10}, x_{20}, \xi_{0}\right)\right|_{\left.\operatorname{graph} T\right|_{\mathcal{R}}}\right)$

where $\beta_{3}(\cdot, \cdot)$ is a locally exponential class- $\mathcal{K} \mathcal{L}$ function and

$\left.\operatorname{graph} T\right|_{\mathcal{R}}=\left\{\left.\left(\left(x_{1}, x_{2}\right), \xi\right) \in \operatorname{graph} \tau\right|_{\mathcal{A}} \times \mathbb{R}^{\bar{p}}: \xi=T\left(x_{1}, x_{2}\right)\right\}$.

To this purpose, pick any $\bar{x}_{10} \in \mathcal{A} \subset \bar{X}_{1}$ and note that $\left(x_{1}(t), x_{2}(t), \xi(t)\right)$ satisfies

$$
\begin{array}{rlrl}
\dot{x}_{1}(t) & =f_{1}\left(x_{1}(t), x_{2}(t)\right) & x_{1}(0)=x_{10} & \\
\dot{x}_{2}(t) & =f_{2}\left(x_{1}(t), x_{2}(t)\right) & x_{2}(0)=x_{20} & \\
\dot{\xi}(t) & =\bar{\varphi}(\xi(t))+\bar{\psi}(\xi(t))\left[\bar{q}\left(\bar{x}_{1}(t)\right)+v(t)\right] & \xi(0)=\xi_{0}
\end{array}
$$

where

$$
v(t)=q\left(x_{1}(t), x_{2}(t)\right)-\bar{q}\left(\bar{x}_{1}(t)\right)
$$

and $\bar{x}_{1}(t)=\Phi_{\bar{f}_{1}}\left(t, \bar{x}_{10}\right)$. Let $x_{1}^{\star} \in \mathcal{A}$ be such that $\left(x_{1}^{\star}, \tau\left(x_{1}^{\star}\right)\right)$ is the projection of $\left(x_{1}, x_{2}\right)$ on graph $\left.\tau\right|_{\mathcal{A}}$. Since $\mathcal{A}$ is forward invariant, $\bar{x}_{1}(t) \in \mathcal{A}$ and $x_{1}^{\star}(t) \in \mathcal{A}$ for all $t \geq 0, X$ is compact and (58) holds, and $\bar{q}$ is locally Lipschitz, for any initial condition $\left(x_{10}, x_{20}\right) \in X$ of (61) the term $v(t)$ can be bounded as

$$
\begin{aligned}
|v(t)| & =\left|q\left(x_{1}(t), x_{2}(t)\right)-\bar{q}\left(x_{1}^{\star}(t)\right)+\bar{q}\left(x_{1}^{\star}(t)\right)-\bar{q}\left(\bar{x}_{1}(t)\right)\right| \\
& \leq\left|q\left(x_{1}(t), x_{2}(t)\right)-\bar{q}\left(x_{1}^{\star}(t)\right)\right|+\left|\bar{q}\left(x_{1}^{\star}(t)\right)-\bar{q}\left(\bar{x}_{1}(t)\right)\right| \\
& \leq L_{q}\left|\left(x_{1}(t), x_{2}(t)\right)\right|_{\text {graph }\left.\tau\right|_{\mathcal{A}}}+2 \sup _{s \in \mathcal{A}}|\bar{q}(s)| \\
& \leq v_{M}
\end{aligned}
$$

for all $t \geq 0$, where $L_{q}$ is a bound of the Lipschitz constant of $q$ on the forward flow of (56) originated from $X$ and $v_{M}$ a positive constant, both dependent on $X$. Hence, from estimate (57), it follows that $\left.\left|\left(\bar{x}_{1}(t), \xi(t)\right)\right|_{\text {graph }} \bar{T}\right|_{\mathcal{A}}$ is bounded and, since $\bar{x}_{1}(t) \in \mathcal{A}$ and graph $\left.\bar{T}\right|_{\mathcal{A}}$ is compact, $\xi(t)$ is also bounded since $\left(x_{1}(t), x_{2}(t)\right)$ originates from $X \subset \mathcal{D}\left(\left.\operatorname{graph} \tau\right|_{\mathcal{A}}\right)$. This, in turn, implies that the trajectories of (59) originated from $X \times \mathbb{R}^{\bar{p}}$ are also ultimately bounded, namely there exists a compact set $S \subset \mathbb{R}^{n_{1}+n_{2}+\bar{p}}$ such that for any $\Xi \subset \mathbb{R}^{\bar{p}}$ there exists a $T>0$ such that $\forall\left(x_{10}, x_{20}, \xi_{0}\right) \in X \times \Xi$, $\left(x_{1}(t), x_{2}(t), \xi(t)\right) \in S$ for all $t \geq T$.

With the previous result at hand, we prove now (60) by following a Lyapunov approach. First, note that, by defining $p_{1}=$ $\operatorname{col}\left(x_{1}, \xi\right)$, the first and third dynamics of (59) can be rewritten as

$$
\dot{p}_{1}=F_{1}\left(p_{1}\right)+G_{1}\left(p_{1}, x_{2}\right)\left(x_{2}-\tau\left(x_{1}\right)\right)
$$

where $F_{1}\left(p_{1}\right)=\operatorname{col}\left(\bar{f}_{1}\left(x_{1}\right), \bar{\varphi}(\xi)+\bar{\psi}(\xi) \bar{q}_{1}\left(x_{1}\right)\right), G_{1}\left(p_{1}, x_{2}\right)=$ $\operatorname{col}\left(r_{1}\left(x_{1}, x_{2}\right), \bar{\psi}(\xi) r_{2}\left(x_{1}, x_{2}\right)\right)$ in which $r_{1}(\cdot, \cdot)$ and $r_{2}(\cdot, \cdot)$ are properly defined smooth functions. By assumption and by converse Lyapunov results (see [22] Theorem 4, [32]), there exist a continuous function $V_{1}: \mathcal{D}\left(\right.$ graph $\left.\left.\bar{T}\right|_{\mathcal{A}}\right) \rightarrow \mathbb{R}$ and positive numbers $c_{1}, \underline{a}_{1} \leq \bar{a}_{1}$, such that

$$
D^{+} V_{1}\left(p_{1}\right) \leq-c_{1} V_{1}\left(p_{1}\right) \quad \forall p_{1} \in \mathcal{D}\left(\left.\operatorname{graph} \bar{T}\right|_{\mathcal{A}}\right)
$$


where $D^{+}\left(p_{1}\right)$ denotes the Dini's derivative at $p_{1}$ defined as

$$
D^{+} V_{1}\left(p_{1}\right):=\lim _{h \rightarrow 0^{+}} \sup \frac{1}{h}\left[V_{1}\left(\phi_{F_{1}}\left(p_{1}, h\right)\right)-V_{1}\left(p_{1}\right)\right]
$$

and

$$
\underline{a}_{1}\left|p_{1}\right|_{\left.\mathrm{graph} \bar{T}\right|_{\mathcal{A}}}^{2} \leq V_{1}\left(p_{1}\right) \leq \bar{a}_{1}\left|p_{1}\right|_{\left.\mathrm{graph} \bar{T}\right|_{\mathcal{A}}}^{2}
$$

$\forall p_{1}:\left(x_{1}, x_{2}, \xi\right) \in S$. Similarly, by letting $p_{2}=\operatorname{col}\left(x_{1}, x_{2}\right)$ and by rewriting (56) as $\dot{p}_{2}=F_{2}\left(p_{2}\right)$, it turns out that there exist a locally Lipschitz function $V_{2}: \mathcal{D}\left(\right.$ graph $\left.\left.\tau\right|_{\mathcal{A}}\right) \rightarrow \mathbb{R}$ and positive numbers $c_{2}, \underline{a}_{2} \leq \bar{a}_{2}$, such that

$$
D^{+} V_{2}\left(p_{2}\right) \leq-c_{2} V_{2}\left(p_{2}\right) \quad \forall p_{2} \in \mathcal{D}\left(\left.\operatorname{graph} \tau\right|_{\mathcal{A}}\right)
$$

where

$D^{+} V_{2}\left(p_{2}\right):=\lim _{h \rightarrow 0^{+}} \sup (1 / h)\left[V_{2}\left(\phi_{F_{2}}\left(p_{2}, h\right)\right)-V_{2}\left(p_{2}\right)\right]$ and

$$
\underline{a}_{2}\left|p_{2}\right|_{\text {graph }\left.\tau\right|_{\mathcal{A}}}^{2} \leq V_{2}\left(p_{2}\right) \leq \bar{a}_{2}\left|p_{2}\right|_{\text {graph }\left.\tau\right|_{\mathcal{A}}}^{2}
$$

$\forall p_{2}:\left(x_{1}, x_{2}, \xi\right) \in S$. Furthermore, note that there exists a positive $\bar{\tau}$ such that

$$
\left|x_{2}-\tau\left(x_{1}\right)\right| \leq \bar{\tau}\left|\left(x_{1}, x_{2}\right)\right|_{\left.\operatorname{graph} \tau\right|_{\mathcal{A}}}
$$

$\forall\left(x_{1}, x_{2}\right):\left(x_{1}, x_{2}, \xi\right) \in S$. As a matter of fact, given $\left(x_{1}, x_{2}, \xi\right) \in S$, let $\bar{x}_{1} \in \mathcal{A}$ be such that $\left|x_{1}-\bar{x}_{1}, x_{2}-\tau\left(\bar{x}_{1}\right)\right|=$ $\left|\left(x_{1}, x_{2}\right)\right|_{\text {graph }\left.\tau\right|_{\mathcal{A}}}$. As $\left|x_{1}-\bar{x}_{1}\right| \leq\left|\left(x_{1}, x_{2}\right)\right|_{\text {graph }\left.\tau\right|_{\mathcal{A}}}$ and $\left|x_{2}-\tau\left(\bar{x}_{1}\right)\right| \leq\left.\left|\left(x_{1}, x_{2}\right)\right|_{\text {graph }} \tau\right|_{\mathcal{A}}$, and denoting by $\bar{\tau}^{\prime}$ an upper bound of the Lipschitz constant of $\tau$ on $S$, it turns out that

$$
\begin{aligned}
\left|x_{2}-\tau\left(x_{1}\right)\right| & =\left|x_{2}-\tau\left(\bar{x}_{1}\right)+\tau\left(\bar{x}_{1}\right)-\tau\left(x_{1}\right)\right| \\
& \leq\left|x_{2}-\tau\left(\bar{x}_{1}\right)\right|+\left|\tau\left(\bar{x}_{1}\right)-\tau\left(x_{1}\right)\right| \\
& \leq\left|\left(x_{1}, x_{2}\right)\right|_{\left.\operatorname{graph} \tau\right|_{\mathcal{A}}}+\bar{\tau}^{\prime}\left|x_{1}-\bar{x}_{1}\right| \\
& \leq\left(1+\bar{\tau}^{\prime}\right)\left|\left(x_{1}, x_{2}\right)\right|_{\left.\operatorname{graph} \tau\right|_{\mathcal{A}}} \\
& :=\bar{\tau}\left|\left(x_{1}, x_{2}\right)\right|_{\left.\operatorname{graph} \tau\right|_{\mathcal{A}}}
\end{aligned}
$$

for all $\left(x_{1}, x_{2}, \xi\right) \in S$, namely (66) holds. Consider now the candidate Lyapunov function $V\left(x_{1}, x_{2}, \xi\right)=V_{1}\left(p_{1}\right)+\beta V_{2}\left(p_{2}\right)$ for system (59) with $\beta>0$. By (63) and (65), there exist positive numbers $\underline{a} \leq \bar{a}$ (dependent on $\beta$ ) such that

$$
\begin{aligned}
& \underline{a}\left|\left(x_{1}, \xi\right)\right|_{\left.\operatorname{graph} \bar{T}\right|_{\mathcal{A}}}^{2}+\underline{a}\left|\left(x_{1}, x_{2}\right)\right|_{\left.\operatorname{graph} \tau\right|_{\mathcal{A}}}^{2} \\
& \quad \leq V\left(x_{1}, x_{2}, \xi\right) \\
& \quad \leq \bar{a}\left|\left(x_{1}, \xi\right)\right|_{\left.\operatorname{graph} \bar{T}\right|_{\mathcal{A}}}^{2}+\bar{a}\left|\left(x_{1}, x_{2}\right)\right|_{\left.\operatorname{graph} \tau\right|_{\mathcal{A}} .}^{2}
\end{aligned}
$$

By (62), (64), (65) and (66), and by the fact that $G_{1}\left(p_{1}, x_{2}\right)$ is locally Lipschitz, it turns out that there exists a $\beta^{\star}>0$ such that for all $\beta \geq \beta^{\star}$ and for all $\left(x_{1}, x_{2}, \xi\right) \in S$

$$
D^{+} V\left(x_{1}, x_{2}, \xi\right) \leq-c V\left(x_{1}, x_{2}, \xi\right)
$$

where $D^{+} V\left(x_{1}, x_{2}, \xi\right)$ is the Dini's derivative of $V$ computed at $\left(x_{1}, x_{2}, \xi\right)$ along the solutions of (59) and $c$ is a positive constant. Combining the previous facts with by (58), standard arguments yields (60) with $\beta_{3}(\cdot, \cdot)$ a locally exponential class- $\mathcal{K} \mathcal{L}$ function. This, in turn, proves the proposition with the functions $(\varphi, \psi, \gamma)$ associated to the triplet $\left(\operatorname{col}\left(f_{1}\left(x_{1}, x_{2}\right), f_{2}\left(x_{1}, x_{2}\right)\right), q\left(x_{1}, x_{2}\right)\right.$, graph $\left.\left.\tau\right|_{\mathcal{A}}\right)$ in the definition of LER given by $(\bar{\varphi}, \bar{\psi}, \bar{\gamma})$.

\section{REFERENCES}

[1] A. N. Atassi and H. K. Khalil, "A separation principle for the stabilization of a class of nonlinear systems," IEEE Trans. Autom. Control, vol. 44, no. 9, pp. 1672-1687, Sep. 1999.

[2] A. Bacciotti, "Linear feedback: The local and potentially global stabilization of cascade systems," in Proc. IFAC Symp. Nonlin. Control Syst. Design, 1992, vol. 2, pp. 21-25.

[3] C. I. Byrnes and A. Isidori, "Asymptotic stabilization of minimumphase nonlinear systems," IEEE Trans. Autom. Control, vol. AC-36, no. 10, pp. 1122-1137, Oct. 1991.

[4] C. I. Byrnes and A. Isidori, "Bifurcation analysis of the zero dynamics and the practical stabilization of nonlinear minimum-phase systems," Asian J. Control, vol. 4, no. 2, pp. 171-185, Jun. 2002.

[5] C. I. Byrnes and A. Isidori, "Limit sets, zero dynamics and internal models in the problem of nonlinear output regulation," IEEE Trans. Autom. Control, vol. AC-48, no. 10, pp. 1712-1723, Oct. 2003.

[6] C. I. Byrnes and A. Isidori, "Nonlinear internal models for output regulation," IEEE Trans. Autom. Control, vol. 49, no. 12, pp. 2244-2247, Dec. 2004.

[7] C. I. Byrnes, F. Celani, and A. Isidori, "Omega-limit sets for a class of nonlinear systems that are semiglobally practically stabilized," Int. J. Robust Nonlin. Control, vol. 15, pp. 315-333, 2005.

[8] F. Esfandiari and H. Khalil, "Output feedback stabilization of fully linearizable systems," Int. J. Control, vol. 56, pp. 1007-1037, 1992.

[9] N. Fenichel, "Geometric singular perturbation theory for ordinary differential equations," J. Diff. Equations, vol. 31, pp. 53-93, 1979.

[10] R. Freeman and P. Kokotović, Robust Nonlinear Control Design: StateSpace and Lyapunov Techniques. Boston, MA: Birkhäuser, 1996.

[11] J. P. Gauthier and I. Kupka, Deterministic Observation Theory and Applications. Cambridge, U.K.: Cambridge Univeraity Press, 2001.

[12] R. Hermann and A. J. Krener, "Nonlinear controllability and observability," IEEE Trans. Autom. Control, vol. AC-22, no. 5, pp. 728-740, 1977.

[13] J. Huang and C. F. Lin, "On a robust nonlinear servomechanism problem," IEEE Trans. Autom. Control, vol. 39, no. 7, pp. 1510-1513, Jul. 1994.

[14] J. Huang, Nonlinear Output Regulation Theory and Applications, ser. Advances in Design and Control. Philadelphia, PA: SIAM, 2004.

[15] A. Isidori, Nonlinear Control Systems II. New York: Springer Verlag, 1999.

[16] Z. P. Jiang, A. R. Teel, and L. Praly, "Small-gain theorem, gain assignment and applications," in Proc. 33rd IEEE Conf. Decision Control, 1994, pp. 3444-3449.

[17] Z. P. Jiang, Control of Interconnected Nonlinear Systems: A SmallGain Viewpoint, ser. Lecture Notes in Control and Information Sciences. Berlin, Germany: Springer, 2004.

[18] I. Kanellakopoulos, P. V. Kokotovic, and A. S. Morse, "A toolkit for nonlinear feedback design,” Syst. Control Lett., vol. 18, pp. 83-92, 1992.

[19] I. Karafyllis and J. Tsinias, "Non-uniform in time ISS and the small-gain theorem," IEEE Trans. Autom. Control, vol. 49, no. 2, pp. 196-216, Feb. 2004.

[20] I. Karafyllis, "Applications of Nonuniform on time robust global asymptotic output stability to robust partial state feedback stabilization," Syst. Control Lett., vol. 54, no. 10, 2005.

[21] L. Marconi and Isidori, "A unifying approach to the design of nonlinear output regulators," in Advances in Control Theory and Applications, ser. Lecture Notes in Control and Information Sciences, C. Bonivento, A. Isidori, L. Marconi, and C. Rossi, Eds. Berlin, Germany: Springer Verlag, 2007.

[22] L. Marconi, L. Praly, and A. Isidori, "Output stabilization via nonlinear Luenberger observers," SIAM J. Control Optim., vol. 45, no. 6, pp. 2277-2298, 2007.

[23] L. Marconi and L. Praly, "Essential and redundant internal models in nonlinear output regulation," in Analysis and Design of Nonlinear Control Systems, A. Astolfi and L. Marconi, Eds. Berlin, Germany: Springer Verlag, 2007.

[24] L. Marconi and L. Praly, "Uniform practical output regulation," IEEE Trans. Autom. Control, vol. 53, no. 5, pp. 1184-1202, May 2008.

[25] F. Mazenc, L. Praly, and W. P. Dayawansa, Global Stabilization by Output Feedback: Examples and Counterexamples vol. 23, no. 2, pp. 119-125, 1994.

[26] H. K. Khalil, Nonlinear Systems, 2nd ed. New York: McMillan, 1996. 
[27] A. Pavlov, N. van de Wouw, and H. Nijmeijer, Uniform Output Regulation of Nonlinear Systems a Convergent Dynamics Approach. Boston, MA: Birkhauser, 2005.

[28] A. R. Teel and L. Praly, "Tools for semiglobal stabilization by partial state and output feedback," SIAM J. Control Optim., vol. 33, pp. 1443-1485, 1995.

[29] A. R. Teel and L. Praly, "Global stabilizability and observability imply semi-global stabilizability by output feedback," Syst. Control Lett., vol. 22, pp. 313-325, 1994.

[30] A. R. Teel, "A nonlinear small gain theorem for the analysis of control systems with saturations," IEEE Trans. Autom. Control, vol. 41, no. 9, pp. 1256-1270, Sep. 1996.

[31] A. R. Teel, L. Moreau, and D. Nesic, "A unified framework for input-tostate stability in systems with two time scales," IEEE Trans. Autom. Control, vol. 48, no. 9, pp. 1526-1544, Sep. 2003.

[32] T. Yoshizawa, Stability Theory and the Existence of Periodic Solutions and Almost Periodic Solutions. New York: Springer Verlag, 1975.

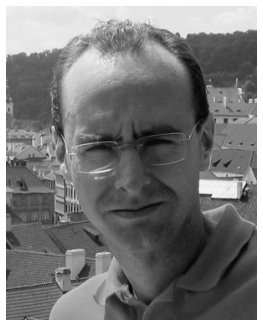

Lorenzo Marconi (S'98-M'99) received the M.S. and $\mathrm{Ph} . \mathrm{D}$. degrees in electrical engineering from the University of Bologna, Bologna, Italy, in 1995 and 1998, respectively.

Since 1995, he has been with the Department of Electronics, Computer Science and Systems, University of Bologna. In 1999, he became an Assistant Professor and has been an Associate Professor since January 2005 . He has held visiting positions at various academic/research international institutions. He is co-author of more than 80 technical publications on the subject of linear and nonlinear feedback design published on international journals, books, and conference proceedings. . His current research interests include nonlinear control, output regulation, control of autonomous vehicles, fault detection and isolation, fault tolerant control.

Dr. Marconi received the "Outstanding Application Paper Award" in 2005 from the International Federation of Automatic Control (IFAC) for a co-authored paper published in Automatica. He is member of the IEEE Control System Society, of the Control System Society Conference Editorial Board, and of IFAC Technical Committees on "Nonlinear Control Systems" and "Safety and Supervision in Technical Processes."

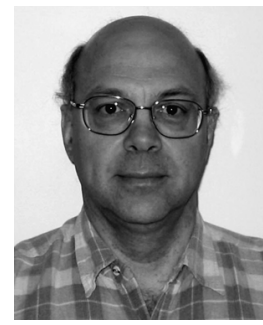

Laurent Praly (M'07) received the Ph.D. degree from École Nationale Supérieure des Mines de Paris, Paris, France, in 1976.

After working in industry for three years, in 1980 he joined the Centre Automatique et Systèmes at École des Mines de Paris. From July 1984 to June 1985, he spent a sabbatical year as a Visiting Assistant Professor in the Department of Electrical and Computer Engineering, University of Illinois at Urbana-Champaign. Since 1985, he has continued at the Centre Automatique et Systèmes where he served as Director for two years. In 1993, he spent a quarter at the Institute for Mathematics and its Applications, University of Minnesota, Minneapolis, where he was an Invited Researcher. His main interest is in feedback stabilization of controlled dynamical systems under various aspects-linear and nonlinear, dynamic, output, under constraints, with parametric or dynamic uncertainty, disturbance attenuation or rejection-on these topics he is contributing both on the theoretical aspect with many academic publications and the practical aspect with applications in power systems, mechanical systems, aerodynamical, and space vehicles.

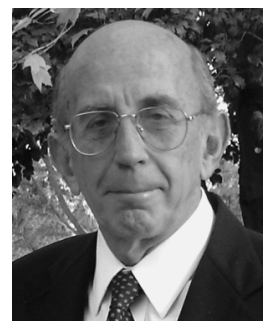

Alberto Isidori (M'80-SM'85-F'87) was born in Rapallo, Italy, in 1942. He received the Ph.D. degree in electrical engineering from the University of Rome, Rome, Italy, in 1965.

Since 1975, he has been Professor of automatic control with the University of Rome. Since 1989 , he has also been affiliated with the Department of Systems Science and Mathematics at Washington University, St. Louis, MO. He has held visiting positions at various academic/research institutions which include the University of Illinois at Urbana-Champaign, the University of California at Berkeley, the ETH, Zürich, Switzerland, and the NASA-Langley research center. He is the author of several books, including Nonlinear Control Systems (New York: Springer Verlag, 1985, 1989 and 1995); Nonlinear Control Systems II (New York: Springer Verlag, 1999). $\mathrm{He}$ is author of more than 80 articles on archival journals, of 16 book chapters and more than 100 papers on refereed conference proceedings, for a large part on the subject of nonlinear feedback design. He is also also Editor/co-Editor of 16 volumes of Conference proceedings. His research interests are primarily focused on mathematical control theory and control engineering.

Dr. Isidori received the G.S.Axelby Outstanding Paper Award in 1981 and in 1990, the Automatica Best Paper Award in 1991 and 2005, the "Georgio Quazza Medal" from the IFAC for "pioneering and fundamental contributions to the theory of nonlinear feedback control" in 1996, the first "Ktesibios Award" from the Mediterranean Control Association, in 2000, and the "Bode Lecture Prize" from the Control Systems Society of IEEE in 2001. 